\title{
Spinor Geometry and General Field Theory
}

\section{G. SZEKERES}

\author{
Communicated by V. HlavatÝ \& R. E. MacKenzie
}

Introduction. The development of geometrical (unified) field theories in the past forty years has been dominated by the idea of generalized metrics and vector connections; spinors have played a comparatively subordinate role. Although spin connection was frequently considered, particularly in connection with the formulation of Dirac's wave equation in general and projective relativity, it was usually treated as a secondary structure superimposed upon the primary metrical structure of the base manifold. ${ }^{1}$

The purpose of the present work is to exploit, more fully than it has been done heretofore, the possibilities of spin connection from the point of view of geometrical field theories and to develop a geometry ${ }^{2}$ whose connection is derived exclusively from the displacement of spinors. There are two circumstances in particular-one geometrical and one physical-which seem to encourage such a development. First, spinors are decidedly more "primitive" geometrical objects than Euclidean vectors, as seen for instance from the fact that, unlike Euclidean rotations, spin transformations represent the full covering group of the orthogonal group of degree $n \geqq 3$. Second, spin connection is known to introduce essentially new elements into the metrical structure which can eventually be interpreted as the geometrical equivalents of the electromagnetic and possibly other physical fields. ${ }^{3}$

The spinor concept to be used is of considerable importance. The spin representation of the 4-dimensional pseudo-orthogonal (Lorentz) group $\mathfrak{L}$ is usually obtained from a (real) Clifford algebra $\Omega$ with four generators; the even elements

\footnotetext{
1 For a bibliography on spinor analysis in general and projective relativity prior to 1953 the reader is referred to [1]. Of references not included there we mention HLAvatý [6] and INGRAHAM [7]. The former deals with spin connection in manifolds with non-symmetrical metric tensor, the latter with spin connection in conformal relativity. INGRAHAM's treatment has a certain affinity to the present one as it is fundamentally based on spin connection.

${ }^{2}$ By a geometry we understand a structure impressed upon a differentiable manifold and consisting of (i) a fibre bundle with suitable differentiability properties and (ii) a connection established by the virtual displacement of the fibre over the manifold.

${ }^{3}$ The fact that spin connection may introduce new elements into the geometrical structure was first noted by Fock [5]. Fock speaks of the "geometrizing" of Dirac's equation; the electromagnetic vector potential appears as part of the spin connection.
} 
of $\Omega$ form a subalgebra $\Omega_{+}$which contains a multiplicative subgroup $\mathscr{L}_{0}$ with the property that $\mathscr{L}_{0} \rightarrow \mathscr{L}$ is a group homomorphism under the correspondence ring multiplication in $\Omega \leftrightarrow$ composition of rotations in $\&$. The kernel of the homomorphism is a subgroup of order 2 consisting of the elements +1 and -1 , and $\mathfrak{L}_{0}$ is a faithful representation of the covering group of 2 . Spinors are by definition vectors in a simple representation space of $\mathfrak{L}_{0}$; they can be identified with elements of $\Omega_{+}$, the action of rotations being expressed as left multiplication by elements of $\mathfrak{L}_{0}$.

If one admits both even and odd elements then the Clifford algebra becomes a natural representation space of the 5 -dimensional pseudo-orthogonal group $\mathscr{K}$; it is therefore tempting to regard $\mathscr{K}$ rather than $\mathscr{L}$ as the fundamental group of spin connection in the sense of Lie-CaRTAN. A still broader outlook is gained if one admits complex coefficients for the Clifford algebra and if inner products are considered. It turns out that the largest continuous and connected group which is contained in $\Omega$ and whose elements leave the inner product of spinors invariant is not $\mathcal{K}_{0}$ (the covering group of $\mathscr{K}$ ) but a group $\mathcal{G}$ which is essentially an extension of $\mathcal{K}_{0}$ by the group of phase transformations (scalars of the form $e^{i \lambda}, \lambda$ real). Because of the fundamental role played by the notion of inner product, it is natural to regard $g$ as the fundamental group of spin connection, and we shall, in fact, do so. The spinors associated with $\mathcal{G}$ have, of course, 16 independent complex components instead of the usual four ${ }^{4}$, but this is not a serious obstacle as it is definitely not our intention to identify the geometrical objects represented by spinors with the probability amplitudes which appear in Dirac's equation. Nevertheless we shall freely accept any hints that may arise from a formal identification of "geometrical" and Dirac spinors.

The proposed geometry can most conveniently be described in the language of fibre bundles. ${ }^{5}$ We assume that the base manifold $R$ has no a priori structure except for the trivial fact that it is a 4-dimensional connected manifold; the bundle space is the carrier of the essential geometrical properties. It consists of two types of fibres, the primary fibre $\Omega$ called the spinor frame and the secondary fibre $\mathfrak{T C}$ called the Minkowski frame. The latter is a 5-dimensional vector frame associated with $\Omega$ in which the rotations of the spinor frame by elements of $\mathcal{G}$ induce pseudo-orthogonal transformations. Since $\mathfrak{T}$ itself does not form part of the "real space" (i.e. of the base manifold $R$ ), there is no harm in using partly imaginary coordinates in $\mathfrak{T}$; this obviates the necessity to distinguish between covariant and contravariant vectors.

The purpose of the Minkowski frame is to translate the geometrical character-

\footnotetext{
${ }^{4}$ 16-component spinors were first considered by EDDINGTon [4], and, in fact, the development of spinor calculus in $\$ 2$ is strongly influenced by EDDINGron's work. At a later stage we shall be led to consider spinors with 64 real components in a Clifford algebra over the skew field of quaternions.

5 The terminology is the one used by STEENRoD in [10]; an exception is the notion of projection which will be used in an entirely different sense. Apart from terminology no specific knowledge of the theory of fibre bundles is required for the understanding of this paper.
} 
istics of spin connection into real quantities pertaining to the manifold $R$. This is accomplished by an operation called projection (\$3) which converts vectors of $\mathfrak{T}$ into real vectors of $R$. Since $\mathfrak{T}$ is 5 -dimensional, projection can be carried out only if $Q$ is temporarily immersed in a 5-dimensional manifold $S$. The auxiliary coordinate however is discarded at the first opportunity and the geometry is ultimately described in terms of real vectors and tensors of $R$. From the projection operator one can derive a symmetrical tensor of second order which takes over the role of the metric tensor, although it is not defined in terms of interval or distance. From the analytical point of view, however, it converts the base space into an ordinary Riemannian manifold ( $\$ 5$ ).

A considerable part of the work is taken up by a description of the postulates of the geometry. They can be divided in two groups. First, there are purely "geometrical" postulates which refer to the spin connection and the embedding process (\$4. (13), §6. (a), (b), (c) and (50)). They have a predominantly "aesthetical" character and are influenced only to a slight extent by empirical considerations. Like Euclid's postulates of 3-space, they can be described in terms of picturesque concepts, but their explicit formulation will be analytical rather than synthetical.

The second group of postulates refers to a spinor field or a "cross-section" of the bundle space which is supposed to be present in the geometrical frame (\$7. (a), (b)). This cross-section is regarded just as much a part of the geometry as the "frame variables" which describe spin connection and projection; its purpose is to create a suitable geometrical object upon which spin connection can operate. But once a cross-section has been introduced, it is necessary to postulate an interaction between spinor field and frame variables to convert the geometry into a tightly knit structure; hence field equations become a necessity and not merely an ad hoc assumption as in the Riemannian formulation of field theories.

The field equations are derived from a 4-dimensional variation principle (§7. (10)); the Lagrangian density which forms the basis of the principle is constructed from the geometrical frame variables and the components of the spinor field by a combined use of physical analogues and mathematical-aesthetical considerations.

Two types of field equations are obtained. Variation of the frame variables gives the field generation equations $(\$ 9 .(F 0)-(F 4)$ and $(G))$; they are nonlinear and determine the "external" (physical) fields generated by a given distribution of the spinor field. Ultimately, the external fields are described by the metric tensor and a complete set of skew tensors of orders $0,1,2,3$ and 4, respectively. The number of independent field equations is the same as the number of independent field components.

Variation of the spinor field gives linear equations of Dirac's type (\$9. (52) or $(M)$ ). They correspond vaguely to the relativistic equations of motion of the electron in quantum mechanics and determine the rate of change of the spinor field under influence of the external fields. 
The analogy with Dirac's equation is very helpful in finding a suitable interpretation of the various field quantities ( $\$ 11)$. For instance, there is no ambiguity in identifying the electromagnetic vector potential $A_{\mu}$ and hence the electromagnetic field strength $F_{\mu \nu}=\partial A_{\mu} / \partial x_{\nu}-\partial A_{\nu} / \partial x_{\mu}$. Similarly one can identify the electromagnetic density as a tensor density $\Re^{\mu \nu}$ whose divergence gives the current density. The two quantities are obtained independently from each other, and they are related only through the field equations. Incidentally, the relation is not MAXweLL's $R_{\mu \nu}=\alpha F_{\mu \nu}$ but-apart from usually quite small non-linear terms $-R_{\mu \nu}=\alpha \square F_{\mu \nu}+$ a tensor whose divergence vanishes. If the vector potential $A_{\mu}$ is normalized by the Lorentz condition $\partial A_{\mu} / \partial x_{\mu}=0$ and $L^{\mu}$ is the current vector, then the wave equation is not $\square A^{\mu}=L^{\mu}$ but $\square \square A^{\mu}=$ $L^{\mu}$.

The physical fields also include a pseudo-scalar and pseudo-tensor meson field and a skew-symmetric "spin-field" of order 3. Except for the pseudoscalar field, they enter the field equations only in the form of non-linear correction terms and are not expected to have any noticeable effects unless the field is very strong. Finally, there is a nowhere vanishing scalar field $A$ whose inverse is interpreted as cosmic time.

The particular form of the Lagrangian requires the existence of an absolute unit of length which is probably related to the classical radius of the electron, but no free dimensionless constants are at our disposal for the fine structure constant and the notorious large number of order $10^{40}$. The position with respect to the fine structure constant is crucial for the whole theory and is tied up with the question (not touched upon in this paper) whether there exist exact solutions of the field equations which would correspond to the electron and other particles. With respect to the second "constant" the position is clear: It is not a constant at all but is proportional to the age of the present epoch. ${ }^{6}$

I am greatly indebted to Professor H. S. Green for some very helpful suggestions at various stages of this work.

\section{Minkowski Frames and Spinor Frames.}

1. The Pseudo-orthogonal Group. ${ }^{7}$ Let $\epsilon_{1}, \cdots, \epsilon_{h}$ be a sequence of numbers which are either +1 or -1 . Consider the group of real transformations

$$
y_{m}=\sum_{n=1}^{h} F_{m n} x_{n}, \quad \operatorname{det}\left[F_{m n}\right]=1
$$

\footnotetext{
${ }^{6}$ In this respect it is particularly reassuring that the mass term in the DiRAc equation turns out to be the recession constant $A$. The idea that a large number of order $10^{40}$ cannot be a constant of nature but must be related to the age of the universe was first suggested by DrRac and taken up later by P. JoRdan. My postulate of isotropic curvature in $\S 6$. is just another variant of JoRDAN's assumption of a variable constant of gravitation.

${ }^{7}$ For the essential properties of the pseudo-orthogonal group see reference [2], in particular Chapter IX.
} 
which carry $\sum_{m} \epsilon_{m} y_{m}{ }^{2}$ into $\sum_{m} \epsilon_{m} x_{m}{ }^{2}$. Under the usual topology of matrices this group has two components, except when $\epsilon_{m}=\epsilon_{n}$ for every $m, n$ in which case the group is connected and is identical with the real proper orthogonal group. In the general case the component containing the identity is itself a group and the abstract group isomorphic to this component will be called the pseudoorthogonal group $\Re=K\left(\epsilon_{1}, \cdots, \epsilon_{h}\right)$ with signature $\left(\epsilon_{1}, \cdots, \epsilon_{h}\right)$. We shall refer to elements of $\mathfrak{K}$ as rotations. The matrices $\mathbf{F}=\left[F_{m n}\right]$ themselves will be called pseudo-orthogonal. They are distinguished by the following property: If

$$
\mathfrak{K}^{+}=\left\{m ; \boldsymbol{\varepsilon}_{m}=+1\right\}, \quad \mathfrak{K}^{-}=\left\{m ; \boldsymbol{\varepsilon}_{m}=-1\right\}
$$

then

$$
\begin{aligned}
& \operatorname{det}\left\{F_{m n} ; m \varepsilon \mathcal{F C}^{+}, n \varepsilon \mathcal{H C}^{+}\right\}>0, \\
& \operatorname{det}\left\{F_{m n} ; m \varepsilon \mathcal{F}^{-}, n \varepsilon \mathcal{F C}^{-}\right\}>0 .
\end{aligned}
$$

The group $\mathfrak{K}$ has an obvious representation by complex orthogonal matrices. Write

$$
j_{m}=1 \text { when } \epsilon_{m}=1, \quad j_{m}=i \text { when } \epsilon_{m}=-1
$$

so that $j_{m}{ }^{2}=\epsilon_{m}$. A suffixed quantity $a_{m}$ will be called real Minkowskian, abbreviated $\operatorname{real}(M)$, if $j_{m} a_{m}$ is real. The definition is extended to quantities depending on several Roman suffixes: $a_{m n \cdots p}$ is called real Minkowskian if $a_{m n} \cdots p$ is real.

Let $\mathrm{J}=\left[J_{m n}\right]$ be defined by

$$
J_{m n}=j_{m} \delta_{m n}
$$

where $\delta_{m n}$ is the Kronecker symbol. If $\mathbf{F}$ is a pseudo-orthogonal matrix then $\mathbf{M}=\mathrm{JFJ}^{-1}$ is clearly orthogonal,

$$
\sum_{p} M_{m p} M_{n p}=\delta_{m n}, \quad \sum_{m} M_{m p} M_{m q}=\delta_{p q}, \quad \operatorname{det} \mathbf{M}=1,
$$

and $M_{m n}$ is real $(M)$. In particular, if $m, n \varepsilon \mathfrak{F C}^{+}$or $m, n \varepsilon \mathfrak{K C}^{-}$then $M_{m n}$ is real and

$$
\begin{aligned}
& \operatorname{det}\left\{M_{m n} ; m \varepsilon \mathcal{H C}^{+}, n \varepsilon \mathcal{H}^{+}\right\}>0, \\
& \operatorname{det}\left\{M_{m n} ; m \varepsilon \mathcal{F C}^{-}, n \varepsilon \mathcal{H C}^{-}\right\}>0 .
\end{aligned}
$$

A matrix $M$ satisfying these conditions will be called a Minkowski matrix (with respect to the signature $\left(\epsilon_{1}, \cdots, \epsilon_{h}\right)$. If $\sigma \varepsilon \Re$ is represented by the pseudoorthogonal matrix $\mathbf{F}=\mathbf{F}(\sigma)$ then $\sigma \rightarrow \mathbf{M}(\sigma)=\mathrm{JF}(\sigma) \mathbf{J}^{-1}$ is a faithful representation of $\mathfrak{K}$ by Minkowski matrices.

$\nVdash$ can also be represented as a Lie group. A set of infinitesimal generators of the group in the Minkowski representation are the matrices

$$
\begin{aligned}
& \mathrm{P}^{(k l)}=\left[P_{m n}^{(k l)}\right], \quad 1 \leqq k<l \leqq h, \\
& P_{m n}^{(k l)}=j_{m} j_{n}\left(\delta_{k m} \delta_{l n}-\delta_{k n} \delta_{l m}\right) .
\end{aligned}
$$


They satisfy the commutator relations

$$
\begin{aligned}
\mathbf{P}^{(k l)} \mathbf{P}^{(m n)}-\mathbf{P}^{(m n)} \mathbf{P}^{(k l)}=\epsilon_{l} \delta_{l m} \mathbf{P}^{(k n)} & -\epsilon_{l} \delta_{l n} \mathbf{P}^{(k m)} \\
& -\epsilon_{k} \delta_{k m} \mathbf{P}^{(l n)}+\epsilon_{k} \delta_{k n} \mathbf{P}^{(l m)}
\end{aligned}
$$

where $\mathbf{P}^{(l k)}=-\mathbf{P}^{(k l)}$ for $k<1$. Consequently every Minkowski matrix has the form

$$
\mathbf{M}=\exp \mathbf{P}, \quad \mathbf{P}=\left[P_{m n}\right], \quad P_{n m}=-P_{m n},
$$

and $P_{m n}$ is $\operatorname{real}(M)$.

Summation convention for repeated roman suffixes: The usual convention will be used, unless the contrary is stated. The convention will never be used in expressions which contain $\epsilon_{m}$ or $j_{m}$.

Minkowski Frames. An $h$-dimensional representation space for $\pi$ is called a Minkowski frame and denoted by $\mathfrak{T}$ if it has the following properties:

$(A)$ Elements of $\mathfrak{T}$ are collections of $h \operatorname{real}(M)$ numbers, called $\mathfrak{T}$-vectors,

$$
\alpha=\left(a_{k} ; k=1, \cdots, h\right), \quad a_{k} \operatorname{real}(M) .
$$

(B) $\mathscr{T}$ admits the rotations $\sigma$ of $\mathscr{K}$ as operators such that

$$
\sigma(\alpha)=\beta=\left(b_{k}\right), \quad b_{k}=M_{k m} a_{m},
$$

where $\left[M_{k m}\right]$ is the Minkowski matrix $M(\sigma)$ representing $\sigma . \mathfrak{T}$ is not an algebraic vector space in the ordinary sense since multiplication by a scalar is not defined. ${ }^{\mathbf{8}}$ In fact the only operations which can be performed on elements of $\mathscr{T}$ are addition and the application of a rotation $\sigma \varepsilon \mathfrak{K}$.

One can also define higher tensors with respect to the Minkowski frame. These are real $(M)$ quantities $R_{k m \cdots n}$ which transform into

$$
S_{k m} \cdots_{n}=M_{k p} M_{m q} \cdots M_{n r} R_{p q} \cdots r
$$

under a rotation $\sigma$. Among the higher tensors we mention the quantity

$$
E_{m_{1}} \cdots m_{h}=j_{1} \cdots j_{h} \operatorname{sig}\left(m_{1} \cdots m_{h}\right)
$$

where $\operatorname{sig}\left(m_{1} \cdots m_{h}\right)=+1$ or -1 according as $\left(m_{1} \cdots m_{h}\right)$ is an even or odd permutation of $(1 \cdots h)$ and 0 otherwise. Clearly $E_{m_{1} \cdots m_{h}}$ is $\operatorname{real}(M)$ and

$$
M_{n_{1} m_{1}} M_{n_{2} m_{2}} \cdots M_{n_{h} m_{h}} E_{m_{1}} \cdots_{m_{h}}=\operatorname{det} M \cdot E_{n_{1} \cdots n_{h}}=E_{n_{1}} \cdots_{n_{h}},
$$

hence $E_{m_{1} \cdots m_{h}}$ is a totally skew-symmetric tensor.

2. Spinors. In various formulas of this and the following sections, repeated suffixes will have to be summed sometimes from 1 to 4 and sometimes from 1 to

\footnotetext{
${ }^{8}$ If complex numbers are admitted then scalar multiplication conflicts with the reality conditions for the vector components. If we only admit real numbers then definition $(B)$ is inadmissible.
} 
5. We make the convention that the letters $k, l, m, \cdots$ always go from 1 to 4 and the letters $a, b, c, \cdots$ from 1 to 5 .

Let $\Gamma_{a}, 1 \leqq a \leqq 5$, be a set of Dirac symbols satisfying

$$
\prod_{a=1}^{5} \Gamma_{a}=1, \quad \Gamma_{a} \Gamma_{b}+\Gamma_{b} \Gamma_{a}=2 \delta_{a b},
$$

and let $\Omega$ denote the (non-commutative) algebra over the complex numbers generated by the $\Gamma_{a}{ }^{9}$ Elements of $\Omega$ can be written in one and only one way in the form

$$
\omega=\alpha+\Gamma_{a} \beta_{a}+\frac{1}{2} \Gamma_{a b} \gamma_{a b}, \quad \Gamma_{a b}=\frac{1}{2 i}\left(\Gamma_{a} \Gamma_{b}-\Gamma_{b} \Gamma_{a}\right)
$$

where $\alpha, \beta_{a}, \gamma_{a b}$ are complex numbers and $\gamma_{b a}=-\gamma_{a b}$. Clearly $\left(\Gamma_{a b}\right)^{2}=1$ if $a \neq b$.

The conjugate of $\omega$ is defined as

$$
\omega^{*}=\alpha^{*}+\Gamma_{a} \beta_{a}^{*}+\frac{1}{2} \Gamma_{a b} \gamma_{a b}^{*}
$$

and the conjugate imaginary of $\omega$ is

$$
\omega^{-}=\alpha^{*}+\Gamma_{a} \beta_{a}^{*}-\frac{1}{2} \Gamma_{a b} \gamma_{a b}^{*}
$$

where $\alpha^{*}$ denotes the conjugate imaginary of $\alpha \cdot{ }^{10}$ One can verify easily that

$$
\left(\omega_{1} \omega_{2}\right)^{*}=\omega_{2}^{*} \omega_{1}^{*}, \quad\left(\omega_{1} \omega_{2}\right)^{-}=\omega_{1}^{-} \omega_{2}^{-} .
$$

$\Omega$ has the important property that it forms a natural representation ring for the four and five dimensional pseudo-orthogonal groups in which the group operation is replaced by ring multiplication. In fact, if $\mathbf{P}^{(a b)}$ denote the infinitesimal generators of the 5-dimensional pseudo-orthogonal group $\mathfrak{K}$, defined by (3), then the ring elements

$$
\Gamma^{(a b)}=\frac{1}{4} i \Gamma_{c d} P^{(a b)}=\frac{1}{2} i j_{a} j_{b} \Gamma_{a b}
$$

(no summation in the last expression!) satisfy the commutator relations

$$
\begin{aligned}
\Gamma^{(a b)} \Gamma^{(c d)}-\Gamma^{(c d)} \Gamma^{(a b)}=\epsilon_{b} \delta_{b c} \Gamma^{(a d)}-\epsilon_{b} \delta_{b d} \Gamma^{(b c)} & \\
& -\epsilon_{a} \delta_{a c} \Gamma^{(b d)}+\epsilon_{a} \delta_{a d} \Gamma^{(b c)}
\end{aligned}
$$

\footnotetext{
- Numbers commute with every element of the algebra and numerical coefficients will be freely written to the left or to the right of the $\Gamma$ 's, whichever way is more convenient. $\Omega$ is a Clifford algebra over the field of complex numbers (also called the algebra of E-numbers by EdDington) and is isomorphic with the ring of $4 \times 4$ complex matrices.

${ }^{10}$ Neither the names nor the notations are standard; for instance M. Rresz [8] uses $\omega^{*}, \tilde{\omega}^{*}$ and $\bar{\omega}$ for what we denote by $\omega^{-}, \omega^{*}$ and $\omega^{+}$, respectively, and calls real what we call self-adjoint.
} 
Therefore the $\Gamma^{(a b)}$ generate a Lie group whose elements have the form ${ }^{11}$

$$
\pi=\exp \left\{\frac{1}{4} i \Gamma_{c d} P_{c d}\right\}, \quad P_{c d}=-P_{d c}
$$

where $P_{c d}$ is $\operatorname{real}(M)$.

Let $\mathfrak{K}_{0}$ denote the group formed by the elements (13). A comparison of (4) and (12) shows that the generators of $\mathfrak{K}_{0}$ satisfy the same commutator relations as the generators of $\mathcal{K}$, hence the two groups are locally isomorphic through the mapping

$$
\exp \left\{\frac{1}{4} i \Gamma_{a b} P_{a b}\right\} \rightarrow \exp \mathbf{P}, \quad \mathbf{P}=\left[P_{a b}\right] .
$$

Nevertheless, the two groups are not isomorphic in the large. In fact, if $a \neq b$ and $\epsilon_{a}=\epsilon_{b}$ so that $P_{a b}$ is real, then the element

$$
\pi_{0}=\exp \left\{\frac{1}{4} i \cdot 2 \pi\left(\Gamma_{a b}-\Gamma_{b a}\right)\right\}=\exp \left\{\pi \Gamma_{a} \Gamma_{b}\right\}=\cos \pi+\Gamma_{a} \Gamma_{b} \sin \pi=-1
$$

is mapped upon the rotation $\exp \left(2 \pi \mathbf{P}^{(a b)}\right)=1$ of $\mathcal{K}$. It can be shown that $\mathcal{K}$ is isomorphic to the quotient group $\mathfrak{K}_{0} /\left\{\pi_{0}\right\}$ where $\left\{\pi_{0}\right\}$ denotes the subgroup consisting of the two elements $\pi_{0}$ and $1 .^{12}$

The above considerations are valid irrespective of the signature of $\mathscr{K}$. Henceforth we shall make the assumption that

$$
\epsilon_{1}=\epsilon_{2}=\epsilon_{3}=-1, \quad \epsilon_{4}=1 .
$$

The value of $\epsilon_{5}$ will not be fixed until much later.

An argument similar to the one above shows that the elements

$$
\begin{array}{cl}
\pi=\exp \left\{\frac{1}{4} i \Gamma_{m n} P_{m n}\right\}, & 1 \leqq m \leqq 4, \quad 1 \leqq n \leqq 4, \\
P_{n m}=-P_{m n}, & P_{m n} \operatorname{real}(M)
\end{array}
$$

form a Lie group $\mathfrak{L}_{0}$, a subgroup of $\mathfrak{K}_{0}$, which is locally isomorphic to the Lorentz group $\&$ (i.e. to the 4-dimensional pseudo-orthogonal group with signature (15)) through the mapping

$$
\exp \left\{\frac{1}{4} i \Gamma_{m n} P_{m n}\right\} \rightarrow \exp \mathbf{P} .
$$

Again $\mathscr{L}$ is isomorphic to $\mathscr{L}_{0} /\left\{\pi_{0}\right\}$.

We may regard $\Omega$ as a vector space which admits $\mathfrak{K}_{0}$ as an operator domain. In other words, we may regard the elements of $\mathscr{K}_{0}$ as rotations applied to the $\Omega$-frame. There are several types of transformation laws which must be considered in this connection.

$\eta$ is called an invariant element of $\Omega$ if it remains unchanged under rotations of the $\Omega$-frame.

$\Psi \varepsilon \Omega$ is called a spinor if under the influence of a rotation $\sigma \varepsilon \mathcal{K}_{0}$, represented

\footnotetext{
$11 \exp \omega$ converges for every $\omega \varepsilon \Omega$. This follows $e . g$. from the existing isomorphism and homeomorphism between $\Omega$ and the ring of $4 \times 4$ complex matrices. The statement expressed by (13) follows from the second fundamental theorem of LrE.

12 These properties are well known, e.g. reference [2], chapters VII and VIII.
} 
by the element $\pi$ according to (13), it transforms into $\pi \Psi$. A dual spinor $\Psi^{+} \varepsilon \Omega$ transforms into $\Psi^{+} \pi^{-1}$ by the same rotation.

Finally $\omega \varepsilon \Omega$ is called an operator if under the rotation $\pi$ it is carried into $\pi \omega \pi^{-1}$.

It follows from these definitions that if $\eta$ is an invariant element, $\Psi$ a spinor, $\Psi^{+}$a dual spinor and $\omega$ an operator, then

$$
\begin{aligned}
& \Psi \eta \text { and } \omega \Psi \text { are spinors, } \\
& \eta \Psi^{+} \text {and } \Psi^{+} \omega \text { are dual spinors. }
\end{aligned}
$$

Operators and invariant elements can therefore be interpreted as linear transformations of the spinor space. If, in particular, the operator has the form (13) then it is called a pseudo-orthogonal transformation.

The adjoint of any element $\omega$ of $\Omega$ is defined as

$$
\omega^{+}=\Gamma \omega^{*} \Gamma
$$

where $\Gamma=\Gamma_{45}$ if $j_{5}=1, \Gamma=\Gamma_{4}$ if $j_{5}=i$. Clearly

$$
\left(\omega_{1} \omega_{2}\right)^{+}=\omega_{2}^{+} \omega_{1}^{+}
$$

and

$$
\left(\omega^{-}\right)^{+}=\left(\omega^{+}\right)^{-}
$$

by (10).

The definition is consistent with the notation for dual spinors. In fact, if $\Psi$ is a spinor then its adjoint is a dual spinor. For,

$$
\Gamma \Gamma_{a} \Gamma=-\epsilon_{5} \epsilon_{a} \Gamma_{a}, \quad \Gamma \Gamma_{a b} \Gamma=\epsilon_{a} \epsilon_{b} \Gamma_{a b}
$$

and

$$
P_{a b}^{*}=\epsilon_{a} \epsilon_{b} P_{a b} \quad \text { if } \quad P_{a b} \text { is } \operatorname{real}(M),
$$

hence $\Gamma\left(\Gamma_{a b} P_{a b}\right) \Gamma=\Gamma_{a b} P_{a b}^{*}$. It follows that

$$
\begin{aligned}
{\left[\exp \left\{\frac{1}{4} i \Gamma_{a b} P_{a b}\right\}\right]^{+} } & =\Gamma\left[\exp \left\{\frac{1}{4} i \Gamma_{a b} P_{a b}\right\}\right]^{*} \Gamma \\
& =\Gamma\left[\exp \left\{-\frac{1}{4} i \Gamma_{a b} P_{a b}^{*}\right\}\right] \Gamma \\
& =\exp \left[-\frac{1}{4} i \Gamma \Gamma_{a b} P_{a b}^{*} \Gamma\right] \\
& =\exp \left\{-\frac{1}{4} i \Gamma_{a b} P_{a b}\right\},
\end{aligned}
$$

i.e.

$$
\pi^{+}=\pi^{-1}
$$

for a rotation $\pi$. Hence by (18)

$$
(\pi \Psi)^{+}=\Psi^{+} \pi^{+}=\Psi^{+} \pi^{-1}
$$

which proves that $\Psi^{+}$is a dual spinor. 
The transformation of operators has a simple explicit expression in terms of the coefficients. If $\omega$ and $\pi$ are given by (7) and (13) respectively, then

$$
\pi \omega \pi^{-1}=\alpha+\Gamma_{a} M_{a b} \beta_{b}+\frac{1}{2} \Gamma_{a b} M_{a c} M_{b d} \gamma_{c d}
$$

where $\mathbf{M}=\left[M_{a b}\right]=\exp \mathbf{P}$ is, by (5), a 5-dimensional Minkowski matrix satisfying the conditions (2).

We can prove (23) either by verifying it for the particular macroscopic rotations

$$
\mathbf{P}=t \mathbf{P}^{(c d)}, \quad t \text { real, }
$$

or, more simply still, by verifying it for infinitesimal rotations. Let

$$
\begin{aligned}
M_{a b} & =\delta_{a b}+\epsilon P_{a b}+O\left(\epsilon^{2}\right), \\
\pi & =1+\frac{1}{4} i \epsilon \Gamma_{c d} P_{c d}+O\left(\epsilon^{2}\right) .
\end{aligned}
$$

Neglecting terms of order $\epsilon^{2}$ and using the relations

$$
\begin{aligned}
\Gamma_{c d} \Gamma_{a}-\Gamma_{a} \Gamma_{c d} & =\frac{2}{i}\left(\Gamma_{c} \delta_{a d}-\Gamma_{d} \delta_{a c}\right), \\
\Gamma_{c d} \Gamma_{a b}-\Gamma_{a b} \Gamma_{c d} & =\frac{2}{i}\left(\Gamma_{b d} \delta_{a c}-\Gamma_{a d} \delta_{b c}-\Gamma_{b c} \delta_{a d}+\Gamma_{a c} \delta_{b d}\right)
\end{aligned}
$$

we get

$$
\begin{aligned}
& \left(1+\frac{1}{4} i \epsilon \Gamma_{c d} P_{c d}\right)\left(\alpha+\Gamma_{a} \beta_{a}+\frac{1}{2} \Gamma_{a b} \gamma_{a b}\right)\left(1-\frac{1}{4} i \epsilon \Gamma_{c d} P_{c d}\right) \\
& \quad=\alpha+\Gamma_{a} \beta_{a}+\epsilon \Gamma_{a} P_{a b} \beta_{b}+\frac{1}{2} \Gamma_{a b} \gamma_{a b}+\frac{1}{2} \epsilon \Gamma_{a b}\left(P_{a c} \gamma_{c b}+P_{b d} \gamma_{a d}\right)
\end{aligned}
$$

which is precisely the relation to be proved. The general statement follows from the existing isomorphism between $\mathfrak{K}_{0}$ and $\mathfrak{K}$.

(23) shows that $\beta_{a}$ transforms like a vector and $\gamma_{a b}$ like a skew tensor in a certain 5-dimensional Minkowski frame. Let us associate with $\Omega$ a 5 -dimensional Minkowski frame $\mathfrak{T}$ which has the property that whenever a rotation (13) is carried out in $\Omega$ then the vectors of $M$ are rotated by the associated rotation $\mathbf{M}=\exp \mathbf{P}$. It follows from (23) that if $\beta_{a}$ is a vector in the associated Minkowski frame and $\gamma_{a b}$ is a skew tensor, then $\Gamma_{a} \beta_{a}$ and $\frac{1}{2} \Gamma_{a b} \gamma_{a b}$ are operators and

$$
\Gamma_{a} \beta_{a} \Psi, \quad \frac{1}{2} \Gamma_{a b} \gamma_{a b} \Psi
$$

are spinors.

$\omega$ is called self-adjoint if $\omega^{+}=\omega$. If $\beta_{a}$ is a vector and $\gamma_{a b}$ a skew tensor in IT then

$$
i j_{5}^{*} \Gamma_{a} \beta_{a} \text { and } \frac{1}{2} \Gamma_{a b} \gamma_{a b}
$$

are self-adjoint operators. This follows immediately from (20) and (21). 
Reflection. If $\omega$ is given by ( 7$)$ then

$$
\Gamma_{5} \omega \Gamma_{5}=\alpha-\Gamma_{m} \beta_{m}+\Gamma_{5} \beta_{5}+\frac{1}{2} \Gamma_{m n} \gamma_{m n}-\Gamma_{m 5} \gamma_{m 5} .
$$

Thus under the influence of $\Gamma_{5}$ the $\mathfrak{T}$-frame suffers the reflection

$$
\beta_{m} \rightarrow-\beta_{m}, \quad \beta_{5} \rightarrow \beta_{5} .
$$

We shall regard reflection as an admissible transformation of the $\Omega$ and $\mathfrak{T}$ frames. Under reflection $\Psi$ transforms into $\Gamma_{5} \Psi$ and $\Psi^{+}$into $\left(\Gamma_{5} \Psi\right)^{+}=\Psi^{+} \Gamma_{5}^{+}=$ $-\Psi^{+} \Gamma_{5}$.

Inner Product. The quarterspur of an element $\omega \varepsilon \Omega$ is defined as

$$
q \mathrm{~s} \omega=\alpha
$$

where $\omega$ is given by (7). It has the following obvious properties:

$$
\mathrm{qs}\left(\omega_{1}+\omega_{2}\right)=\mathrm{qs} \omega_{1}+\mathrm{qs} \omega_{2}, \quad \mathrm{qs}(c \omega)=c \mathrm{qs} \omega
$$

for a number $c$,

$$
q \mathrm{qs}\left(\omega_{1} \omega_{2}\right)=\mathrm{qs}\left(\omega_{2} \omega_{1}\right), \quad \mathrm{qs}\left(\omega^{*}\right)=(\mathrm{qs} \omega)^{*} .
$$

The inner product of an ordered pair of spinors $\Psi_{1}$ and $\Psi_{2}$ is defined as

$$
\left\langle\Psi_{1} \Psi_{2}\right\rangle=\mathrm{qs}\left(\Psi_{1}^{+} \Psi_{2}\right)=\mathrm{qs}\left(\Gamma \Psi_{1}^{*} \Gamma \Psi_{2}\right)=\mathrm{qs}\left(\Psi_{1}^{*} \Gamma \Psi_{2} \Gamma\right) .
$$

It is always a number and must not be confused with the ring product $\Psi_{1} \Psi_{2}$ which is a general element of $\Omega$. The following properties can be verified easily:

$$
\begin{gathered}
\left\langle\left(\Psi_{1}+\Psi_{2}\right) \Psi\right\rangle=\left\langle\Psi_{1} \Psi\right\rangle+\left\langle\Psi_{2} \Psi\right\rangle \\
\left\langle\Psi\left(\Psi_{1}+\Psi_{2}\right)\right\rangle=\left\langle\Psi \Psi_{1}\right\rangle+\left\langle\Psi \Psi_{2}\right\rangle \\
\left\langle\Psi_{1}\left(c \Psi_{2}\right)\right\rangle=c\left\langle\Psi_{1} \Psi_{2}\right\rangle \\
\left\langle\Psi_{2} \Psi_{1}\right\rangle=\left\langle\Psi_{1} \Psi_{2}\right\rangle^{*} .
\end{gathered}
$$

For any operator $\omega$

$$
\left\langle\Psi_{1}\left(\omega \Psi_{2}\right)\right\rangle=\left\langle\left(\omega^{+} \Psi_{1}\right) \Psi_{2}\right\rangle .
$$

The expression in (30) will be denoted $\left\langle\Psi_{1} \omega \Psi_{2}\right\rangle$.

From (28) and (30) we have

$$
\left\langle\left(c \Psi_{1}\right) \Psi_{2}\right\rangle=c^{*}\left\langle\Psi_{1} \Psi_{2}\right\rangle
$$

and from (29) and (30)

$$
\left\langle\Psi_{1} \omega \Psi_{2}\right\rangle=\left\langle\Psi_{2} \omega^{+} \Psi_{1}\right\rangle^{*}
$$

If therefore $\omega$ is self-adjoint then the expression $\frac{1}{2}\left\{\left\langle\Psi_{1} \omega \Psi_{2}\right\rangle+\left\langle\Psi_{2} \omega \Psi_{1}\right\rangle\right\}$, and in particular $\langle\Psi \omega \Psi\rangle$, is a real number. For the former expression we shall use the notation $\frac{1}{2}\left\langle\Psi_{1} \omega \Psi_{2}+\Psi_{2} \omega \Psi_{1}\right\rangle$. 
(26) is not the only possible definition of the inner product which has the properties (27)-(30). In fact, if $\zeta$ is any fixed self-adjoint element then

$$
\left\langle\Psi_{1} \Psi_{2}\right\rangle=\operatorname{qs}\left(\zeta \Psi_{1}^{+} \Psi_{2}\right)
$$

is a suitable definition. We mention that although (26) is the simplest definition with our notations, it is not identical with the one commonly used in quantum mechanics which corresponds to

$$
\left\langle\Psi_{1} \Psi_{2}\right\rangle=\operatorname{qs}\left(\Psi_{1}^{*} \Gamma \Psi_{2}\right)=\operatorname{qs}\left(\Gamma \Psi_{1}^{+} \Psi_{2}\right) .
$$

The value of the inner product (whichever definition is adopted) is independent of the orientation of $\Omega$, i.e. is invariant with respect to rotations $\pi \varepsilon \mathcal{K}_{0}$. For

$$
\left\langle\left(\pi \Psi_{1}\right)\left(\pi \Psi_{2}\right)\right\rangle=\left\langle\Psi_{1} \pi^{+} \pi \Psi_{2}\right\rangle=\left\langle\Psi_{1} \Psi_{2}\right\rangle
$$

by (22). Also it remains invariant with respect to phase transformations, i.e. to multiplying every $\Psi$ by a common phase factor $e^{i \lambda}, \lambda$ real. This remark suggests that the group $\mathfrak{K}_{0}$ should be extended to a larger group $\mathcal{G}^{13}$, called the spinor group, which also includes phase transformations. $\mathcal{G}$ consists of all transformations

$$
\rho=\exp \left\{i \lambda+\frac{1}{4} i \Gamma_{a b} P_{a b}\right\},
$$

$\lambda$ real, $P_{a b} \operatorname{real}(M), P_{b a}=-P_{a b}$, and we have the result: The inner product is invariant under transformations of $\mathcal{G}$.

Reflection changes the sign of the inner product:

$$
\left\langle\left(\Gamma_{5} \Psi_{1}\right)\left(\Gamma_{5} \Psi_{2}\right)\right\rangle=\left\langle\Psi_{1} \Gamma_{5}^{+} \Gamma_{5} \Psi_{2}\right\rangle=-\left\langle\Psi_{1} \Psi_{2}\right\rangle .
$$

We have seen that the rotation $\Psi \rightarrow \pi_{0} \Psi=-\Psi$ induces the identity rotation in the associated Minkowski frame $\mathfrak{T}$. Now $\pi_{0}=\exp (\pi i)$ is a particular phase transformation, and we make the agreement that every phase transformation shall induce the identity rotation in $\mathfrak{N}$, that is, (33) shall induce $\mathbf{M}=\exp \mathbf{P}$, $\mathbf{P}=\left[P_{a b}\right]$. The convention cannot lead to any contradiction, and, in fact, it is the only possible one to make, since phase transformations belong to the central of $\mathcal{G}$, whereas the only element in the central of $\mathscr{K}$ is the identity. If $\mathcal{C}$ denotes the central of $\mathcal{G}$, i.e. the subgroup of phase transformations, then $\mathscr{K}$ is isomorphic to $\mathrm{G} / \mathrm{C}$.

The elements

$$
\sigma=\exp \left\{i \lambda+\frac{1}{4} i \Gamma_{m n} P_{m n}\right\},
$$

consisting of a phase transformation and a rotation of $\mathfrak{L}_{0}$, form a subgroup FC $\subset$ G. Operators of the form

$$
\tau=\exp \left\{\Gamma_{5} \alpha\right\}=\cosh \alpha+\Gamma_{5} \sin \mathrm{h} \alpha, \quad \alpha \text { real, }
$$

have the property that rotations of $\mathfrak{H C}$ leave them invariant. In fact, $\Gamma_{5} \Gamma_{m n}=$ $\Gamma_{m n} \Gamma_{5}$ hence $\sigma \tau=\tau \sigma$ for every $\sigma \varepsilon \mathcal{H}$. Also

${ }^{13}$ The spinor group is not to be confused with the Clifford group of Chevallex [3], p. 49. 


$$
\tau^{+}=\Gamma \exp \left\{\Gamma_{5} \alpha^{*}\right\} \Gamma=\exp \left\{-\Gamma_{5} \alpha\right\}=\tau^{-1}
$$

which shows that the inner product of spinors is invariant under transformations $\tau$. Thus the operators (35) behave very much like phase transformations, and we shall call them phase transformations of the second kind. Clearly the transformations (35) form a group; it will be denoted by $\propto$. Again we agree that if a phase transformation of the second kind is carried out in $\Omega$, this induces the identity in $\mathfrak{T}$. The condition is compatible with our earlier conventions provided that the group of reorientations is restricted to $\mathfrak{F}$.

An invariant element $\eta$ is called unitary if

$$
\eta^{+} \eta=1 .
$$

If $\eta$ is unitary and $\Psi_{1}, \Psi_{2}$ are arbitrary spinors then

$$
\left\langle\left(\Psi_{1} \eta\right)\left(\Psi_{2} \eta\right)\right\rangle=\left\langle\Psi_{1} \Psi_{2}\right\rangle
$$

provided that the definition (26) is adopted for the inner product. For

$$
\left\langle\left(\Psi_{1} \eta\right)\left(\Psi_{2} \eta\right)\right\rangle=\mathrm{qs}\left(\eta^{+} \Psi_{1}^{+} \Psi_{2} \eta\right)=\mathrm{qs}\left(\Psi_{1}^{+} \Psi_{2}\right)=\left\langle\Psi_{1} \Psi_{2}\right\rangle .
$$

\section{Manifolds with Spin Connection.}

3. Projection. A topological space $R$ is called an h-dimensional manifold if each point $p$ has a neighborhood which can be parametrized by $h$ real coordinates $x_{v}$ in a rectangle $\mathrm{e}^{14}$

$$
x: \quad \alpha_{\nu}<x_{\nu}<\beta_{\nu}, \quad \nu=1, \cdots, h .
$$

We say that $p$ is covered by the frame $x$. Since we fix our attention on local properties rather than topology in the large, we shall usually assume that the region under consideration can be covered by a single frame $x$. If

$$
\text { Y: } \alpha_{\nu}^{\prime}<y_{\nu}<\beta_{\nu}^{\prime}, \quad \nu=1, \cdots, h
$$

is a second frame covering the same region, it will be further assumed that the functions $y_{\nu}=y_{\nu}\left(x_{1}, \cdots, x_{h}\right)$ have partial derivatives of any desired order and

$$
\frac{\partial(y)}{\partial(x)}=\frac{\partial\left(y_{1}, \cdots, y_{h}\right)}{\partial\left(x_{1}, \cdots, x_{h}\right)} \neq 0
$$

throughout the region. The class of admissible frames is uniquely fixed by these conditions, provided that at least one frame of the class is known.

A set of (real, complex or $\Omega$-valued) functions $A^{\nu}(x)=A^{\nu}\left(x_{1}, \cdots, x_{h}\right)$, $\nu=1, \cdots, h$, is said to be contravariant in $\nu$ if they transform into

$$
B^{\mu}(y)=\sum_{\nu=1}^{h} \frac{\partial y_{\mu}}{\partial x_{\nu}} A^{\nu}(x), \quad \mu=1, \cdots, h
$$

\footnotetext{
${ }^{14}$ It is naturally assumed that the parametrization is a homeomorphism of the neighbourhood of $p$ into the Euclidean rectangle $X$.
} 
when the coordinates are changed; $A_{\nu}(x)$ is called covariant in $\nu$ if the transformation law is

$$
B_{\mu}(y)=\sum_{\nu=1}^{h} \frac{\partial x_{\nu}}{\partial y_{\mu}} A_{\nu}(x), \quad \mu=1, \cdots, h .
$$

Whenever a Greek index is affixed to a function on $R$, it is understood (and in every given case it must be checked) that the function transforms covariantly or contravariantly with the $x$-frame, according to the position of the index.

An $x$-tensor field (or briefly a tensor) is a function with one or more Greek suffixes; in the former case it will be called an $X$-vector. A tensor at a point $p=(x)$ is the value which a tensor takes at $(x)$. A scalar is a function which has no Greek suffixes at all, hence its value at any particular point does not depend on the $\mathcal{X}$-frame. A density is a function which, besides being transformed like a tensor (or scalar), is multiplied by $\partial(x) / \partial(y)$.

Summation convention for repeated Greek suffixes: The usual convention is used with the proviso that one index must appear as a subscript and the other as a superscript.

Starting from a four-dimensional manifold $R$, we shall construct now a fibre bundle which has $\mathbb{R}$ for its base space. As a first step, we attach to the generic point $p \varepsilon R$ a spinor frame $\Omega=\Omega(p)$ and its associated Minkowski frame $\mathscr{T}(p)$ and establish a relation between $\mathfrak{T}(p)$ and $R$ by projecting vectors of $\mathscr{T}(p)$ into the manifold $R$. Since $\mathscr{T}$ is 5 -dimensional, the projection can most conveniently be described if $R$ is immersed in a 5 -dimensional manifold $S$ with an auxiliary coordinate $x_{0}$. We choose the $x$-frame $\left(x_{0}, x_{1}, x_{2}, x_{3}, x_{4}\right)=\left(x_{0}, x\right)$ in $S$ so that $(0, x)$ shall represent points in $R$ and the direction of the $x_{0}$-axis shall everywhere be perpendicular to $R$, in a sense explained below. To emphasize the exceptional position of the auxiliary coordinate, we shall always write out the suffix 0 explicitly and shall not include it among the Greek summation suffixes.

Projection is described as a univalent linear mapping

$$
\begin{aligned}
A_{\mu} & =\alpha_{a} g_{a \mu}, \quad A_{0}=\alpha_{a} g_{a 0}, & \mu & =1, \cdots, 4, \\
\alpha_{a} & =g_{a}{ }^{\mu} A_{\mu}+g_{a}{ }^{0} A_{0}, & a & =1, \cdots, 5
\end{aligned}
$$

of the vectors of $\mathscr{T}(p)$ upon the real covariant $X$-vectors at $p$. Note that 0 is always a Greek suffix and 5 a roman suffix. Clearly

$$
\begin{aligned}
& g_{a}{ }^{\mu} g_{a \nu}=\delta_{\nu}{ }^{\mu}, \quad g_{a}{ }^{\mu} g_{a 0}=g_{a}{ }^{0} g_{a \nu}=0, \\
& g_{a}{ }^{0} g_{a 0}=1, \quad g_{a \mu} g_{b}{ }^{\mu}+g_{a 0} g_{b}{ }^{0}=\delta_{a b} .
\end{aligned}
$$

The assumed "perpendicularity" of the $x_{0}$-axis is expressed by the equations

$$
g_{a \mu} g_{a 0}=0, \quad g_{a}{ }^{\mu} g_{a}{ }^{0}=0 .
$$

More specifically, we require that, with a suitable orientation of the $\Omega$ and $\mathfrak{T}$ frames and using a suitable unit in the $x_{0}$-direction, 
(3) $\quad g_{n}{ }^{0}=g_{n 0}=g_{5}{ }^{\mu}=g_{5 \mu}=0, \quad n=1, \cdots, 4, \quad \mu=1, \cdots, 4$,

$$
\begin{gathered}
g_{5}{ }^{0}=\zeta_{0} j_{5}, \quad g_{50}=\zeta_{0} j_{5}^{*}, \quad \zeta_{0}=+1 \text { or }-1, \\
\mathfrak{g}=\frac{1}{i} \operatorname{det}\left[g_{m \mu}\right] \neq 0 .
\end{gathered}
$$

We shall call an $X$-frame normal if, with a suitable orientation of $\Omega$ (hence of IT), conditions (3), (4), (5) can be satisfied; and the orientation itself which fulfills these conditions will be called normal. Using a normal frame and orientation we have

$$
g_{n}{ }^{\mu} g_{n \nu}=\delta_{\nu}{ }^{\mu}, \quad g_{m}{ }^{\mu} g_{n \mu}=\delta_{m n},
$$

and equations (1) take the form

$$
\begin{array}{ll}
A_{\mu}=\alpha_{m} g_{m \mu}, & \alpha_{m}=g_{m}{ }^{\mu} A_{\mu}, \\
A_{0}=\zeta_{0} j_{5}^{*} \alpha_{5}, & \alpha_{5}=\zeta_{0} j_{5} A_{0} .
\end{array}
$$

The quantities $g_{m \mu}=g_{m \mu}(x)$ are functions of the coordinates, but $\zeta_{0}$ is supposed to have the same value at all points covered by $x$. Evidently $g_{m \mu}$ is covariant and $g_{m}{ }^{\mu}$ contravariant in $\mu$, and both are $\mathfrak{T}$-vectors with respect to $m$ and have $\operatorname{real}(M)$ values. The quantity

$$
\mathfrak{g}=\frac{1}{i} \operatorname{det}\left[g_{m \mu}\right]
$$

is a real scalar density since

$$
\operatorname{det}\left[g_{m \mu} \frac{\partial x_{\mu}}{\partial y_{\nu}}\right]=\frac{\partial(x)}{\partial(y)} \operatorname{det}\left[g_{m \mu}\right] .
$$

We call $A_{\mu}$ the covariant projection of $\alpha_{m}$ in $R$. There is also a contravariant projection of $\alpha_{m}$ in $Q$ defined by $A^{\mu}=g_{m}{ }^{\mu} \alpha_{m}, \alpha_{m}=g_{m \mu} A^{\mu}$. Generally one can change any contravariant or covariant Greek suffix of a tensor into a roman suffix and conversely:

$$
\begin{aligned}
& T_{n}^{\cdots \cdots}=g_{n \mu} T^{\mu \cdots} \cdots, \quad T^{\mu \cdots} \cdots=g_{n}{ }^{\mu} T_{n} \cdots, \\
& T_{\mu}^{\cdots} \cdots=g_{n \mu} T_{n}^{\cdots} \cdots, \quad T_{n}^{\cdots} \cdots=g_{n}{ }^{\mu} T_{\mu} \cdots . \\
& T_{5}^{\cdots} \cdots=\zeta_{0} j_{5} T_{0}^{\cdots} \cdots=\zeta_{0} j_{5}^{*} T^{0} \cdots \text {. }
\end{aligned}
$$

In particular,

$$
\begin{array}{ll}
T_{\mu}^{\cdots}=g_{\mu \nu} T_{\cdots}^{\nu \cdots,} & T^{\mu}=g^{\mu \nu} T_{\nu}^{\cdots}, \\
T_{0}^{\cdots} \cdots=g_{00} T_{\cdots}^{0 \cdots} & T_{\cdots}^{0} \cdots=g^{00} T_{0}^{\cdots} \cdots
\end{array}
$$

where

$$
\begin{aligned}
& g_{\mu \nu}=g_{n \mu} g_{n \nu}, \quad g^{\mu \nu}=g_{m}{ }^{\mu} g_{m}{ }^{\nu}, \\
& g_{00}=g^{00}=\epsilon_{5}, \quad g_{0 \mu}=g^{0 \mu}=0 .
\end{aligned}
$$


Thus $g_{\mu \nu}$ behaves like the metric tensor of Riemannian geometry; it is real and has the symmetry $g_{\mu \nu}=g_{\nu \mu}$. Clearly $\left(\operatorname{det}\left[g_{n \nu}\right]\right)^{2}=\operatorname{det}\left[g_{\mu \nu}\right]$, hence

$$
|\mathfrak{g}|=\left(-\operatorname{det}\left[g_{\mu \nu}\right]\right)^{\frac{1}{2}} \text {. }
$$

This shows incidentally that $|\mathfrak{g}|$ is invariant under rotations of the $\mathfrak{T}$ frame. ${ }^{15}$ The same result can be obtained from the skew tensor $E_{a b c d e}$ constructed at the end of section I.1. We have

$$
\begin{aligned}
& E_{0 \mu \nu \rho \sigma}=g_{a 0} g_{b \mu} g_{c \nu} g_{d \rho} g_{e \sigma} E_{a b c d e}=\zeta_{0} \mathfrak{g} \operatorname{sig}(\mu \nu \rho \sigma), \\
& E^{0 \mu \nu \rho \sigma}=-\zeta_{0} \epsilon_{5} \mathfrak{g}^{-1} \operatorname{sig}(\mu \nu \rho \sigma),
\end{aligned}
$$

and $\mathfrak{g} E^{0 \mu \nu \rho \sigma}$ is, apart from a \pm sign, the well known $\epsilon$-density.

In the future we shall only admit normal frames and orientations. Consequently, we shall only allow those transformations of the $X$-frame which belong to one of the following types:

(10) Transformations which at the points of $\propto$ do not involve $x_{0}$ at all.

(11) Transformations of the form $x_{0}=\lambda y_{0}$ where $\lambda$ is a positive constant.

(12) The transformation $x_{0}=-y_{0}$ which reverses the direction of $x_{0}$.

Tensors which under the last transformation reverse their sign are called pseudo-tensors (respectively pseudo-scalars). Thus the quantity $\zeta_{0}$ in (4) is a pseudo-scalar and $E_{0 \mu \nu \rho \sigma}$ in (9) is a totally skew pseudo-tensor.

A transformation of the type (11) will be called a gauge transformation by $\lambda$; it corresponds to a change of unit on the $x_{0}$-axis to its $\lambda$-fold. To avoid a conflict with formula (4), we assume that a gauge transformation induces a corresponding change of the unit vector in $\mathfrak{T}(p)$ in the fixed proportion $\lambda$ at every $p$. Clearly such a change of gauge in $\mathscr{T}$ does not affect the components of Minkowski transformations. On the other hand, it obviously affects the projection vectors $g_{n \mu}, g_{n}{ }^{\mu}$. Let us call, for the sake of present discussion, the transformation

$$
y_{\mu}=\lambda^{-1} x_{\mu}, \quad \mu=1, \cdots, 4,
$$

a scale transformation of the $x$-frame. Then the most plausible convention concerning the effect of a gauge transformation upon the projection vectors is this: We require that if the gauge is changed by $\lambda$ in $\mathscr{T}$, and simultaneously the scale is changed by $\lambda$ in $x$, then the components of the projection vectors shall remain unchanged.

Now $g_{n}{ }^{\mu}$ is contravariant and $g_{n \mu}$ is covariant in $\mu$, hence a scale transformation of $X$ by $\lambda$ carries $g_{n}{ }^{\mu}$ into $\lambda^{-1} g_{n}{ }^{\mu}$ and $g_{n \mu}$ into $\lambda g_{n \mu}$. Therefore in order to comply with the previous postulate we have to assume that a gauge transformation by $\lambda$ carries $g_{n}{ }^{\mu}$ into $\lambda g_{n}{ }^{\mu}$ and $g_{n \mu}$ into $\lambda^{-1} g_{n \mu}$. As a consequence $g_{\mu \nu}$ and $g^{\mu \nu}$ are gauge dependent, the first one receiving a factor $\lambda^{-2}$, the second one a factor $\lambda^{2}$.

${ }^{15} \mathfrak{g}$ itself may change its sign. If, as will be done further below, we restrict the admissible rotations of $\mathfrak{T}$ to the connected component of the subgroup of normal rotations, i.e. rotations which do not conflict with the normality conditions, then $\mathfrak{g}$ itself remains invariant. 
We say a tensor is of gauge index $j$ if, under a gauge transformation by $\lambda$, it obtains the factor $\lambda^{i}$. Thus

$$
\begin{aligned}
& g_{\mu \nu} \text { is a tensor of gauge index }-2, \\
& g_{\mu \nu} \text { is a tensor of gauge index } 2, \\
& \mathfrak{g} \text { is a scalar density of gauge index }-4 .
\end{aligned}
$$

It follows that raising a Greek suffix in a tensor raises its gauge index by 2 . Forming a density from a tensor by multiplying it with $\mathfrak{g}$ lowers its gauge index by 4 .

4. Spin Connection. The fundamental operation which establishes spin connection is congruent displacement; it refers to a virtual transfer of spinors of $\Omega(p)$ into neighbouring points $p+d p=(x+d x)$. The effect of the displacement is an infinitesimal transformation by an element of the spinor group $g$ with respect to the spinor frame $\Omega(p+d p)$. It is expressed by the differential form

$$
\delta \Psi=\left(i A_{\mu} d x^{\mu}+\frac{1}{4} i \Gamma_{a b} S_{a b \mu} d x^{\mu}\right) \Psi
$$

where $A_{\mu}$ is real and covariant in $\mu, S_{a b \mu}=-S_{b a \mu}$ is real $(M)$ and covariant in $\mu$ for every fixed $a, b$.

Formula (13) is an analytical expression of the postulate that the fundamental group of space (in the sense of LIE-CARTAN) is $\mathrm{G}$. It is tacitly assumed that the spinor frames have a suitable orientation, i.e. that there are no discontinuities in their orientations with respect to the topology of the base space. In fact we shall require more than mere continuity; we postulate that, with a suitable orientation of the $\Omega(p)$ and $\mathscr{T}(p)$ and a suitable choice of the class of admissible $x$-frames, the functions $A_{\mu}(x), S_{a b \mu}(x)$ and $g_{n \mu}(x)$ have partial derivatives of any desired order.

A spinor field is a function $\Psi(x)$ over $\mathbb{R}$ with values in $\Omega(p)$. The spin derivatives of $\Psi(x)$ and its adjoint $\Psi^{+}(x)$ are defined as

$$
\begin{gathered}
\Psi_{/ \mu}=\Psi_{, \mu}-\left(i A_{\mu}+\frac{1}{4} i \Gamma_{a b} S_{a b \mu}\right) \Psi \\
\Psi_{/ \mu}^{+}=\left(\Psi_{/ \mu}\right)^{+}=\Psi^{+}{ }_{, \mu}+\Psi^{+}\left(i A_{\mu}+\frac{1}{4} i \Gamma_{a b} S_{a b \mu}\right)
\end{gathered}
$$

where $\mu$ after a comma denotes partial differentiation with respect to $x_{\mu}$.

$\Psi_{/ \mu}$ is a spinor field provided that the quantities $A_{\mu}$ and $S_{a b \mu}$ have suitable transformation laws under reorientation. A reorientation $\sigma$ is, by definition, a function with values in the group $\mathfrak{H}$, (definition I. (34),

$$
\sigma(x)=\exp \left\{i \lambda(x)+\frac{1}{4} i \Gamma_{m n} P_{m n}(x)\right\}
$$

where the functions $\lambda(x)$ and $P_{m n}(x)$ have partial derivatives of any desired order. Owing to the fact that the mixed components $P_{m 5}$ vanish, normal frames remain normal after reorientation. 
Let $\widetilde{A}_{\mu}, \widetilde{S}_{a b \mu}$ be the components of spin connection after reorientation, and write

$$
\begin{aligned}
& M_{55}=1, \quad M_{m 5}=M_{5 m}=0, \\
& \mathbf{M}=\exp \mathbf{P}, \quad \mathbf{M}=\left[M_{m n}\right], \quad \mathbf{P}=\left[P_{m n}\right] .
\end{aligned}
$$

The reorientation (15) carries $\Psi_{/ \mu}$ into

$$
\begin{aligned}
& \left(\exp \left\{i \lambda+\frac{1}{4} i \Gamma_{m n} P_{m n}\right\} \Psi\right)_{, \mu}-\left(i \widetilde{A}_{\mu}+\frac{1}{4} i \Gamma_{a b} \widetilde{S}_{a b \mu}\right) \exp \{\} \Psi \\
& =\exp \{\}\left\{\Psi_{, \mu}-\left(i \tilde{A}_{\mu}-i \lambda_{, \mu}+\frac{1}{4} i \Gamma_{m n} M_{p m} M_{q n} \widetilde{S}_{p q \mu}\right.\right. \\
& \left.\left.+\frac{1}{2} i \Gamma_{m 5} M_{p m} \widetilde{S}_{p 5 \mu}-\frac{1}{4} i \Gamma_{m n} M_{p m} M_{a n} P_{p q, \mu}\right) \Psi\right\}
\end{aligned}
$$

by II. (23) and by the formula

$$
\left(\exp \left\{\frac{1}{4} i \Gamma_{m n} P_{m n}\right\}\right)_{, \mu}=\exp \left\{\frac{1}{4} i \Gamma_{m n} P_{m n}\right\} \cdot \frac{1}{4} i \Gamma_{r s} M_{p r} M_{p s, \mu} \cdot
$$

To prove (17), note that for

$$
\begin{gathered}
\pi=\exp \left\{\frac{1}{4} i \Gamma_{m n} P_{m n}\right\}=\exp \left\{\sum_{m<n} \Gamma^{(m n)} p_{m n}\right\}, \quad P_{m n}=j_{m} j_{n} p_{m n} \\
\pi^{-1} \pi_{, \mu}=\sum_{r<s} f_{r s} \Gamma^{(r s)}
\end{gathered}
$$

where the coefficients $f_{r s}$ depend only on the $p_{m n}, p_{m n, \mu}$ and the structural constants of the Lie group. Therefore also

$$
\mathbf{M}^{-1} \mathbf{M}_{, \mu}=\sum_{r<s} f_{r s} \mathbf{P}^{(r s)}
$$

and

$$
\left(\mathbf{M}^{-1} \mathbf{M}_{, \mu}\right)_{r s}=-\left(\mathbf{M}^{-1} \mathbf{M}_{, \mu}\right)_{s r}=j_{r} j_{s} f_{r s} \quad \text { for } \quad r<s
$$

Hence

$$
\pi^{-1} \pi_{, \mu}=\frac{1}{4} i \Gamma_{r s} j_{r} j_{s} f_{r s}=\frac{1}{4} i \Gamma_{r s}\left(\mathbf{M}^{-1} \mathbf{M}_{, \mu}\right)_{r s},
$$

which is precisely the formula (17).

Expression (16) is equal to

$$
\exp \left\{i \lambda+\frac{1}{4} i \Gamma_{m n} P_{m n}\right\} \Psi_{/ \mu}
$$

provided that

$$
\begin{gathered}
\tilde{A}_{\mu}=A_{\mu}+\lambda_{, \mu}, \\
\widetilde{S}_{m n \mu}=M_{m p} M_{n q} S_{p q \mu}+M_{m p, \mu} M_{n p}, \\
\widetilde{S}_{m 5 \mu}=M_{m p} S_{p 5 \mu} .
\end{gathered}
$$

These are the required transformation laws. Since $S_{m 5 \mu}$ transforms like an Tr-vector,

$$
-T_{0 \rho \mu}=T_{\rho 0 \mu}=\zeta_{0} j_{5}^{*} g_{m \rho} S_{m 5 \mu}
$$

is a real orientation-invariant pseudo-tensor of gauge index -1 . 
Under reflection, $A_{\mu}$ and $S_{m n \mu}$ remain unchanged, and $S_{m 5 \mu}$ transforms into $-S_{m 5 \mu}$. The tensor $T_{\rho \rho \mu}$ itself is invariant under reflection since $g_{m \rho}$ transforms into $-g_{m \rho}$.

From (14) we obtain by straightforward calculation

$$
\Psi_{/ \nu / \mu}-\Psi_{/ \mu / \nu}=i F_{\mu \nu} \Psi+\frac{1}{4} i \Gamma_{m n} R_{m n \mu \nu} \Psi+\frac{1}{2} i \Gamma_{m 5} R_{m 5 \mu \nu} \Psi
$$

where

$$
\begin{gathered}
F_{\mu \nu}=A_{\mu, \nu}-A_{\nu, \mu}, \\
R_{a b \mu \nu}=S_{a b \mu, \nu}-S_{a b \nu, \mu}+S_{a c \mu} S_{c b \nu}-S_{a c \nu} S_{c b \mu} .
\end{gathered}
$$

From (18) it is clear that the phase curvature tensor $F_{\mu \nu}$ is orientation invariant. Also it is gauge invariant (is of gauge index 0 ) since $A_{\mu}$ is obviously so by definition. ${ }^{16} R_{a b \mu \nu}$ is real $(M)$, has the symmetries

$$
R_{a b \mu \nu}=-R_{b a \mu \nu}=-R_{a b \nu \mu}=R_{b a \nu \mu}
$$

and is covariant in $\mu, \nu$. To find out its transformation law with respect to reorientation, it is more convenient to write (24) in the matrix form

$$
\mathbf{R}_{\mu \nu}=\mathbf{S}_{\mu, \nu}-\mathbf{S}_{\nu, \mu}+\mathbf{S}_{\mu} \mathbf{S}_{\nu}-\mathbf{S}_{\nu} \mathbf{S}_{\mu}
$$

where, for fixed $\mu, \nu, \mathbf{R}_{\mu \nu}=\left[R_{a b \mu \nu}\right]$ and $\tilde{\mathbf{S}}_{\mu}=\left[S_{a b \mu}\right]$. Then (19) and (20) can be written

$$
\widetilde{\mathbf{S}}_{\mu}=\mathbf{M S}_{\mu} \mathbf{M}^{\prime}+\mathbf{M}_{, \mu} \mathbf{M}^{\prime}=\mathbf{M S}_{\mu} \mathbf{M}^{\prime}-\mathbf{M M}_{, \mu}^{\prime}
$$

where $\mathbf{M}^{\prime}$ is the transpose of $\mathbf{M}$, and we get

$$
\begin{aligned}
\tilde{\mathbf{R}}_{\mu \nu}=\left(\mathbf{M S}_{\mu} \mathbf{M}^{\prime}+\right. & \left.\mathbf{M}_{, \mu} \mathbf{M}^{\prime}\right)_{, \nu}-\left(\mathbf{M S}_{\nu} \mathbf{M}^{\prime}+\mathbf{M}_{, \nu} \mathbf{M}^{\prime}\right)_{, \mu} \\
& +\left(\mathbf{M S}_{\mu} \mathbf{M}^{\prime}+\mathbf{M}_{, \mu} \mathbf{M}^{\prime}\right)\left(\mathbf{M S}_{\nu} \mathbf{M}^{\prime}-\mathbf{M M}_{, \nu}^{\prime}\right) \\
& \quad-\left(\mathbf{M S}_{\nu} \mathbf{M}^{\prime}+\mathbf{M}_{, \nu} \mathbf{M}^{\prime}\right)\left(\mathbf{M S}_{\mu} \mathbf{M}^{\prime}-\mathbf{M M}_{, \mu}^{\prime}\right)=\mathbf{M R}_{\mu \nu} \mathbf{M}^{\prime} .
\end{aligned}
$$

This shows that $R_{a b \mu \nu}$ is an $97-$ tensor and

$$
\begin{gathered}
R_{\rho \sigma \mu \nu}=g_{m \rho} g_{n \sigma} R_{m n \mu \nu}, \\
-R_{0 \rho \mu \nu}=R_{\rho 0 \mu \nu}=\zeta_{0} j_{5}^{*} g_{m \rho} R_{m \tilde{\mu} \mu \nu}
\end{gathered}
$$

are real tensors whose components are invariant under reorientations. They are of gauge index -2 since $S_{a b \mu}$ and $R_{a b \mu \nu}$ are gauge invariant. $R_{\rho \sigma \mu \nu}$ is the curvature tensor of the spin connection, it has the obvious symmetries

$$
R_{\rho \sigma \mu \nu}=-R_{\sigma \rho \mu \nu}=-R_{\rho \sigma \nu \mu} .
$$

\footnotetext{
${ }^{16}$ The expression "gauge invariance" is used here in much the same sense as originally proposed by WeYL and EDDington. What is commonly called gauge invariance in present day quantum mechanics is really invariance with respect to phase transformations.
} 
Consequently the only non-trivial contraction of (26) is the Ricci-Einstein tensor

$$
R_{\mu \nu}=g^{\rho \sigma} R_{\rho \mu \nu \sigma}=g_{m}{ }^{\rho} g_{n \mu}\left(S_{m n \nu, \rho}-S_{m n \rho, \nu}+S_{m a \nu} S_{a n \rho}-S_{m a \rho} S_{a n \nu}\right) .
$$

$R_{\mu \nu}$ is a gauge invariant world tensor and is generally not symmetrical in $\mu$ and $\nu$.

5. Riemannian Metric. Spin connection automatically induces a connection for vectors in $\mathfrak{T}(p)$, and the spin derivatives of a vector field $\alpha_{a}$ are given by

$$
\alpha_{a / \nu}=\alpha_{a, \nu}-S_{a b \nu} \alpha_{b} \text {. }
$$

If $A^{0}, A^{\mu}$ are the contravariant projections of $\alpha_{5}, \alpha_{m}$ then the contravariant projection of $\alpha_{m / \nu}$ is

$$
\begin{aligned}
A^{\mu}{ }_{\nu \nu} & =g_{m}{ }^{\mu} \alpha_{m / \nu}=\left(g_{m}{ }^{\mu} \alpha_{m}\right)_{, \nu}-g_{m}{ }^{\mu}{ }_{, \nu} \alpha_{m}-g_{m}{ }^{\mu} S_{m a \nu} \alpha_{a} \\
& =A^{\mu}{ }_{, \nu}+\Gamma^{\mu}{ }_{\nu \nu} A^{\rho}-T^{\mu}{ }_{0 \nu} A^{0}
\end{aligned}
$$

and the corresponding projection of $\alpha_{5 / \nu}$ is

$$
A^{0}{ }_{\nu}=\zeta_{0} j_{5} \alpha_{5 / \nu}=\zeta_{0} j_{5} \alpha_{5, \nu}+\zeta_{0} j_{5} S_{m 5 \nu} \alpha_{m}=A^{0}{ }_{, \nu}-T^{0}{ }_{\rho \nu} A^{\rho}
$$

where

$$
-\Gamma_{\rho \nu}^{\mu}=g_{m}{ }^{\mu} g_{n \rho} S_{m n \nu}+g_{m}{ }^{\mu}{ }_{\nu}{ } g_{m \rho}
$$

and $T_{\mu 0 \nu}$ is the tensor defined in (21).

$\Gamma_{\rho \nu}^{\mu}$ is the nearest approach to what is usually called the affinity of the manifold, but it has no particular significance in spinor geometry. In fact, expressions (31) and (32) are quite artificial and we shall never have any opportunity to use them. The only useful derivatives of world tensors are the ordinary Riemannian invariant (or metrical) derivatives; they are obtained from the Christoffel affinity

$$
\left\{\begin{array}{c}
\mu \\
\rho \nu
\end{array}\right\}=\frac{1}{2} g^{\mu \lambda}\left(g_{\lambda \rho, \nu}+g_{\lambda \nu, \rho}-g_{\rho \nu, \lambda}\right)
$$

in the usual manner, e.g.

$$
\begin{aligned}
A^{\mu}{ }_{; \nu} & =A^{\mu}{ }_{, \nu}+\left\{\begin{array}{c}
\mu \\
\nu \lambda
\end{array}\right\} A^{\lambda}, \quad A_{\mu ; \nu}=A_{\mu, \nu}-\left\{\begin{array}{c}
\lambda \\
\mu \nu
\end{array}\right\} A_{\lambda}, \\
\mathfrak{A}_{; \nu} & =\mathfrak{A}_{, \nu}-\left\{\begin{array}{c}
\lambda \\
\nu \lambda
\end{array}\right\} \mathfrak{U} .
\end{aligned}
$$

Thus from the point of view of real tensors and densities, $R$ will be treated as an ordinary Riemannian manifold with the metric tensor $g_{\mu \nu} .{ }^{17}$ In particular,

${ }^{17}$ In principle one could incorporate the $T$-tensor of equation (42) in the affinity of the real world vectors; this is a matter of definition (and convenience) since the base manifold has no a priori affinity for $\mathcal{X}$-vectors. If this is done then $R$ becomes a general "metrically connected" manifold in the sense of SCHRöDINGER [9], part III. 
we can construct the Riemannian or metrical curvature tensor

$$
G^{\mu}{ }_{\nu \rho \sigma}=-\left\{\begin{array}{c}
\mu \\
\nu \rho
\end{array}\right\}_{, \sigma}+\left\{\begin{array}{c}
\mu \\
\nu \sigma
\end{array}\right\}_{, \rho}+\left\{\begin{array}{c}
\mu \\
\tau \rho
\end{array}\right\}\left\{\begin{array}{c}
\tau \\
\nu \sigma
\end{array}\right\}-\left\{\begin{array}{c}
\mu \\
\tau \sigma
\end{array}\right\}\left\{\begin{array}{c}
\tau \\
\nu \rho
\end{array}\right\}
$$

and the metrical Einstein tensor

$$
G_{\mu \nu}=-\left\{\begin{array}{c}
\lambda \\
\mu \nu
\end{array}\right\}_{, \lambda}+\left\{\begin{array}{c}
\lambda \\
\mu \lambda
\end{array}\right\}_{, \nu}+\left\{\begin{array}{c}
\tau \\
\mu \lambda
\end{array}\right\}\left\{\begin{array}{c}
\lambda \\
\tau \nu
\end{array}\right\}-\left\{\begin{array}{c}
\lambda \\
\tau \lambda
\end{array}\right\}\left\{\begin{array}{c}
\tau \\
\mu \nu
\end{array}\right\} .
$$

They have the well known symmetries

$$
\begin{aligned}
& G_{\mu \nu \rho \sigma}=-G_{\nu \mu \rho \sigma}=-G_{\mu \nu \sigma \rho}=G_{\rho \sigma \mu \nu}, \\
& G_{\mu \nu \rho \sigma}+G_{\mu \rho \sigma \nu}+G_{\mu \sigma \nu \rho}=0, \quad G_{\nu \mu}=G_{\mu \nu} .
\end{aligned}
$$

Since the Christoffel symbols are gauge invariant, the same is true for $G^{\nu}{ }_{\mu \rho \sigma}$ and $G_{\mu \nu}$.

To find out the relation between the two curvature tensors $G^{\mu}{ }_{\nu \rho \sigma}$ and $R_{\nu \rho \sigma}^{\mu}$, write

$$
\begin{gathered}
Q_{m n \nu}=g_{m \rho, \nu} g_{n}{ }^{\rho}-g_{m \mu} g_{n}{ }^{\rho}\left\{\begin{array}{c}
\mu \\
\rho \nu
\end{array}\right\}, \\
S_{m n \nu}=Q_{m n \nu}+T_{m n \nu} .
\end{gathered}
$$

From (8), (34) and (38) one gets by a simple calculation

$$
\begin{aligned}
Q_{m n \nu}=\frac{1}{2} g_{m}{ }^{\rho}\left(g_{n \nu, \rho}-g_{n \rho, \nu}\right)-\frac{1}{2} g_{n}{ }^{\rho}\left(g_{m \nu, \rho}-\right. & \left.g_{m \rho, \nu}\right) \\
& \quad-\frac{1}{2} g_{m}{ }^{\mu} g_{n}{ }^{\rho} g_{r \nu}\left(g_{r \mu, \rho}-g_{r \rho, \mu}\right)
\end{aligned}
$$

which shows that $Q_{n m \nu}=-Q_{m n \nu}$, hence $T_{n m \nu}=-T_{m n \nu}$. Reorientation changes $Q_{m n v}$ into

$$
\left(M_{m p} g_{p \rho}\right)_{, \nu} M_{n q} g_{q}{ }^{\rho}-M_{m p} M_{n q} g_{p \mu} g_{q}{ }^{\rho}\left\{\begin{array}{c}
\mu \\
\rho \nu
\end{array}\right\}=M_{m p} M_{n q} Q_{p q \nu}+M_{m p, \nu} M_{n p},
$$

hence $T_{m n \nu}$ into $M_{m p} M_{n q} T_{p q \nu}$ by (19) and (39). This proves that $T_{m n v}$ is an গT-tensor.

$T_{\rho \nu}^{\mu}=g_{m}{ }^{\mu} g_{n \rho} T_{m n \nu}$ is a real gauge invariant tensor with the obvious symmetry

$$
T_{\mu \rho \nu}=-T_{\rho \mu \nu} \text {. }
$$

(33), (38) and (39) give the relation

$$
-\Gamma_{\rho \nu}^{\mu}=-\left\{\begin{array}{c}
\mu \\
\rho \nu
\end{array}\right\}+T_{\rho \nu}^{\mu} .
$$

Geodesic orientations. We can always choose the $\mathcal{X}$-frame so that at a particular point $p^{18}$

$$
g_{\mu \rho, \nu}=0, \quad\left\{\begin{array}{c}
\mu \\
\rho \nu
\end{array}\right\}=0
$$

\footnotetext{
${ }^{18}$ A proof is given by Schrödinger [9], p. 37.
} 
for every $\mu, \rho, \nu$. Such a frame is called geodesic at $p$. Using a geodesic frame one can choose the orientation of the IT-frame so that

$$
g_{n \mu, \nu}=0 \quad \text { for every } n, \mu, \nu .
$$

In fact, let $y^{\mu}$ be the relative coordinates (in a geodesic $x$-frame) at $p$ and write

$$
M_{m n}=\exp \left\{\frac{1}{2}\left(g_{m}{ }^{\lambda} g_{n \lambda, \nu}-g_{n}{ }^{\lambda} g_{m \lambda, \nu}\right) y^{\nu}\right\} .
$$

This is clearly a Minkowski matrix which becomes the identity at $p$, and we can use $M_{m n}(y)$ for reorientation. Now at $p$

$$
\begin{aligned}
\left(M_{m n} g_{n \mu}\right)_{, \nu} & =g_{m \mu, \nu}+\frac{1}{2}\left(g_{m}{ }^{\lambda} g_{n \lambda, \nu}-g_{n}{ }^{\lambda} g_{m \lambda, \nu}\right) g_{n \mu} \\
& =\frac{1}{2}\left(g_{m \mu, \nu}+g_{m}{ }^{\lambda} g_{n \mu} g_{n \lambda, \nu}\right) \\
& =\frac{1}{2}\left(g_{m \mu, \nu}-g_{m}{ }^{\lambda} g_{n \lambda} g_{n \mu, \nu}\right)=0
\end{aligned}
$$

by (6), which proves the statement.

We shall call an orientation of the Tr-frames geodesic at $p$ if $g_{n \mu, \nu}=0$ in an $x$-frame which is geodesic at $p$. A necessary and sufficient condition for an orientation to be geodesic at $p$ is that

$$
g_{n \mu, \nu}=g_{n v, \mu} \quad \text { for every } n, \mu, \nu
$$

at $p$. For, (44) is a tensor relation which holds in every $\mathcal{X}$-frame if it holds in a certain $x$-frame. But, using a geodesic $X$-frame, (44) certainly holds if the orientation is geodesic. Conversely, suppose (44) to be true, then by (40) $Q_{m n v}=0$ for every $m, n, \nu$, hence, using a geodesic $X$-frame, $g_{m \rho, \nu} g_{n}{ }^{\rho}=0$ by (38), and $g_{m \rho, \nu}=0$ for every $m, \rho, \nu$.

With the help of geodesic frames and orientations we can now prove the relations

$$
\begin{gathered}
R_{\mu \nu \rho \sigma}=G_{\mu \nu \rho \sigma}+T_{\mu \nu \rho ; \sigma}-T_{\mu \nu \sigma ; \rho}+T_{\mu \tau \rho} T_{\nu \sigma}^{\tau}-T_{\mu \tau \sigma} T_{\nu \rho}^{\tau} \\
+T_{\mu 0 \rho} T_{\nu \sigma}^{0}-T_{\mu 0 \sigma} T_{\nu \rho}^{0} \\
R_{0 \sigma \mu \nu}=-R_{\sigma 0 \mu \nu}=T_{0 \sigma \mu ; \nu}-T_{0 \sigma \nu ; \mu}+T_{0 \tau \mu} T_{\sigma \nu}^{\tau}-T_{0 \tau \nu} T_{\sigma \mu}^{\tau} .
\end{gathered}
$$

The proof follows simply from the remark that (45) and (46) are orientationinvariant tensor relations, and they are obviously correct, by (24), (26), (27) and (43), in a geodesic frame and orientation.

For purposes of calculation it will be sometimes convenient to separate out the metrical derivatives of a spinor field:

$$
\Psi_{; \mu}=\Psi_{, \mu}-\frac{1}{4} i \Gamma_{m n} Q_{m n \mu} \Psi .
$$

This is not a spinor with respect to phase transformations, although it behaves 
like a spinor with respect to rotations. Nevertheless it serves a useful purpose, particularly if a transformation of the phase frame is not contemplated.

6. Connection in the Embedding Space. The curvature components (45) and (46) are incomplete inasmuch as there are no components of the type $R_{\mu \nu 0 \sigma}$ or $R_{0 \nu v \sigma}$. The obvious reason is that we have only contemplated a connection in $R$ itself, but not in the embedding space $S$. We shall now try to extend the spin connection into a suitably constructed embedding space so as to obtain the missing curvature components. The extension will be carried out in two steps: First, spin connection will be defined in layers parallel to $R$ and, second, along the $x_{0}$-axis itself.

With respect to the first step, the most obvious thing to do is to assume perfect homogenity, i.e. independence of the components of connection and projection from the position of the layer. An undesirable feature of this assumption is that it puts all the layers on an equal footing and there is nothing to distinguish $Q$ from the other layers. One way out would be to regard $x_{0}$ as a projective coordinate and identify equivalent points of the various layers, but such an attitude would not fit very well with the character of the projection process which definitely requires a 5-dimensional base manifold. We shall therefore adopt a less restrictive standpoint which at the same time reflects sufficiently the exceptional character of the auxiliary dimension; we allow for an isotropic Riemannian curvature in the $x_{0}$-direction. The postulate we are going to formulate amounts to the assumption that the curvature of any surface element through the $x_{0}$-axis at a given point is independent of the orientation of the surface element.

Let us introduce a spinor frame $\Omega(q)$ plus its associated Minkowski frame $\mathscr{T}(q)$ at the generic point $q \varepsilon \mathcal{S}$. Let $\Re(t)$ denote the 4-dimensional hypersurface $x_{0}=t$ so that $R(0)=R$. Then the postulate of homogenity and isotropic curvature takes the following form:

There exist normal $\mathcal{X}$-frames covering an arbitrary $q \varepsilon \delta$ such that, with a suitable orientation of the $\Omega(q)$, the following conditions are fulfilled:

(a) Relations (3) and (4) hold everywhere.

(b) $g_{n \mu}\left(x_{0}, x\right)=g_{n \mu}(0, x) \cos \left(\frac{1}{2} A j_{0} x_{0}\right)$ where $A \neq 0$ is a real scalar of gauge index 1 and $j_{0}$ is either 1 or $i^{19}$

(c) The components of spin connection in $\Omega_{(}(t)$ are such that $A_{\mu}(t, x)=$ $A_{\mu}(0, x), T^{\rho}{ }_{\mu \nu}(t, x)=T_{\mu \nu}^{\rho}(0, x), T^{0}{ }_{\mu \nu}(t, x)=T^{0}{ }_{\mu \nu}(0, x)$.

The new field variable $A$ is a function of the coordinates in $R(0)$ and, of course, has any number of partial derivatives. Since $A \neq 0, A$ is either positive or negative throughout $R$.

Clearly the admissible coordinate transformations (10), (11) and (12) do not conflict with conditions (a), (b) and (c), provided that conditions (10)-(12)

\footnotetext{
${ }^{19} j_{0}=1$ corresponds to spherical and $j_{0}=i$ to hyperspherical curvature. We shall find later that $j_{0}=i$. It is necessary to distinguish clearly between $j_{0}$ and $j_{5}$.
} 
are everywhere fulfilled. On the other hand, the admissible reorientations do not conflict with (a)-(c) if they have the form

$$
\sigma\left(x_{0}, x\right)=\exp \left\{i \lambda(x)+i \chi\left(x_{0}\right)+\frac{1}{4} i \Gamma_{m n} P_{m n}(x)\right\}
$$

where $\lambda(x), P_{m n}(x)$ do not depend on $x_{0}$ and $\chi\left(x_{0}\right)$ is a function of $x_{0}$ alone.

From (a), (b), (c) it would be possible to calculate the components of spin connection in $R(t)$ for every $t$, but this is unnecessary since we are only interested in the components of the curvature tensor at $R(0)$, for which a knowledge of the connection outside the immediate neighbourhood of $R$ is of no importance.

From (b) we get, if the values at $x_{0}=0$ are marked by an asterisk,

$$
\begin{aligned}
g_{n \mu} & =g^{*}{ }_{n \mu}\left(1-\frac{1}{8} A^{2} \epsilon_{0} x_{0}{ }^{2}+O\left(x_{0}{ }^{4}\right)\right), \\
g_{\mu \nu} & =g^{*}{ }_{\mu \nu}\left(1-\frac{1}{4} A^{2} \epsilon_{0} x_{0}{ }^{2}+O\left(x_{0}{ }^{4}\right)\right), \\
\left\{\begin{array}{c}
\mu \\
\rho \nu
\end{array}\right\} & =\left\{\begin{array}{c}
\mu \\
\rho \nu
\end{array}\right\}^{*}+O\left(x_{0}{ }^{2}\right), \\
\left\{\begin{array}{c}
0 \\
\rho \nu
\end{array}\right\} & =\frac{1}{4} A^{2} \epsilon_{0} \epsilon_{5} x_{0} g^{*}{ }_{\nu \rho}+O\left(x_{0}{ }^{2}\right), \\
\left\{\begin{array}{c}
\mu \\
0 \nu
\end{array}\right\} & =-\frac{1}{4} A^{2} \epsilon_{0} x_{0} \delta_{\nu}{ }^{\mu}+O\left(x_{0}{ }^{2}\right), \\
\left\{\begin{array}{c}
0 \\
\nu
\end{array}\right\} & =\left\{\begin{array}{c}
\mu \\
00
\end{array}\right\}=\left\{\begin{array}{c}
0 \\
00
\end{array}\right\}=0,
\end{aligned}
$$

where $\epsilon_{0}=j_{0}{ }^{2}$ and the $O$-symbols refer to $x_{0} \rightarrow 0$. These give, by (38),

$$
\begin{aligned}
& Q_{m n \nu}=Q^{*}{ }_{m n \nu}+O\left(x_{0}{ }^{2}\right), \\
& Q_{m 5 \nu}=\frac{1}{4} \zeta_{0} j_{5} \epsilon_{0} A^{2} x_{0} g^{*}{ }_{m \nu}+O\left(x_{0}{ }^{2}\right),
\end{aligned}
$$

hence, by (39) and (c),

$$
S_{m n \nu}=S_{m n \nu}^{*}+O\left(x_{0}^{2}\right)
$$

and

$$
S_{m 5 \nu}=S^{*}{ }_{m 5 \nu}+\frac{1}{4} \zeta_{0} j_{5} A^{2} \epsilon_{0} x_{0} g^{*}{ }_{m \nu}+O\left(x_{0}{ }^{2}\right) .
$$

With respect to the spin connection along the $x_{0}$-axis, our guiding principle is that such a displacement shall not involve any transformations which would induce rotations in the associated Minkowski frame. Hence we postulate:

The fundamental group of spin connection along the $x_{0}$-axis is the group of phase transformations, $\mathrm{e}$.

Consequently, congruent displacement in the direction of $x_{0}$ is given by

$$
\delta \Psi=i A_{0} d x^{0} \Psi
$$


where $A_{0}$ is a real pseudo-scalar of gauge index 1 . The spin derivative with respect to $x_{0}$ is, accordingly,

$$
\Psi_{/ 0}=\Psi_{, 0}-i A_{0} \Psi, \quad \Psi^{+}{ }_{/ 0}=\Psi^{+}{ }_{0}+\Psi^{+} i A_{0} .
$$

If a reorientation (48) is carried out, then $A_{0}$ transforms into

$$
\tilde{A}_{0}=A_{0}+\chi_{, 0} \text {. }
$$

This follows from

$$
(\sigma \Psi)_{, 0}-i \tilde{A}_{0} \sigma \Psi=\sigma \Psi,{ }_{, 0}+i \chi_{, 0} \sigma \Psi-i \widetilde{A}_{0} \sigma \Psi=\sigma\left(\Psi_{, 0}-i A_{0} \Psi\right) .
$$

In defining $\Psi_{/ 0}$ we have made no hypothesis regarding the dependence of $\Psi$ on $x_{6}$. The most obvious homogeneity conditions would be

$$
\Psi\left(x_{0}, x\right)=\exp \left\{\frac{1}{2} i \zeta_{0} A x_{0}\right\} \Psi(0, x) .
$$

These must be rejected for the reason that any dependence of the form

$$
\Psi\left(x_{0}, x\right)=\exp \left\{\text { if }\left(x_{0}\right)\right\} \Psi(0, x)
$$

can be destroyed by a suitable reorientation $\sigma=\exp (i \chi)$ where $\chi\left(x_{0}\right)=-f\left(x_{0}\right)$. The difficulty can be resolved if we replace the phase factor in (53) by a corresponding phase transformation of the second kind, as defined in I. (35). We call $\Psi$ homogeneous if its $x_{0}$-dependence has the form

$$
\begin{aligned}
\Psi\left(x_{0}, x\right) & =\exp \left\{\frac{1}{2} \Gamma_{5} \zeta_{0} A x_{0}\right\} \Psi(0, x) \\
& =\left\{\cosh \left(\frac{1}{2} \zeta_{0} A x_{0}\right)+\Gamma_{5} \sinh \left(\frac{1}{2} \zeta_{0} A x_{0}\right)\right\} \Psi(0, x) .
\end{aligned}
$$

It is plainly desirable that this should be in harmony with postulate (b); therefore we postulate that the isotropic curvature is hyperspherical,

$$
j_{0}=i, \quad \epsilon_{0}=-1 .
$$

A homogeneous spinor field determines the phase frame uniquely at every $R(t), t \neq 0$, since reorientations of the form $\exp \left\{i \chi\left(x_{0}\right)\right\}$ would conflict with (54). Therefore the admissible reorientations have the form

$$
\sigma\left(x_{0}, x\right)=\sigma(x)=\exp \left\{i \lambda(x)+\frac{1}{4} i \Gamma_{m n} P_{m n}(x)\right\},
$$

and the transformation law for $A_{0}$ becomes

$$
\tilde{A}_{0}=A_{0} \text {. }
$$

Hence $A_{0}$ is a proper pseudo-scalar which is independent of reorientations. Note that the spin derivatives (14) of a homogeneous spinor field are not homogeneous because of the $x_{0}$-dependence of $S_{m 5 \mu}$.

(51) and (54) give for a homogeneous $\Psi$

$$
\Psi_{/ 0}=\left(\frac{1}{2} \Gamma_{5} \zeta_{0} A-i A_{0}\right) \Psi, \quad \Psi^{+}{ }_{10}=\Psi^{+}\left(-\frac{1}{2} \Gamma_{5} \zeta_{0} A+i A_{0}\right) .
$$


Curvature. Let $\Psi$ be an arbitrary spinor field, not necessarily homogeneous. Straightforward calculation gives at $R(0)$, using (14), (49), (51) and (55),

$$
\Psi_{/ \nu / 0}-\Psi_{/ 0 / \nu}=i F_{0 \nu} \Psi+\frac{1}{2} i \Gamma_{m 5} R_{m 50 \nu} \Psi
$$

where

$$
F_{0 \nu}=A_{0, v}
$$

and

$$
R_{m 50 v}=\frac{1}{4} \zeta_{0} j_{5} A^{2} h_{m \nu} .
$$

This gives the following new curvature components:

$$
R_{\mu 00 \nu}=-R_{0 \mu 0 \nu}=\frac{1}{4} A^{2} g_{\mu \nu} .
$$

The components $R_{\mu \nu 0 \sigma}$ are missing because of the normality of coordinates.

The complete Einstein tensor is defined as

$$
R^{*}{ }_{\mu \nu}=R_{\mu \nu}+R_{\mu \nu 0}^{0}
$$

where $R_{\mu \nu}$ is given by (29) and

$$
R_{\mu \nu 0}^{0}=\frac{1}{4} \epsilon_{5} A^{2} g_{\mu \nu} .
$$

The other components of the tensor are, by (24), (27) and (64),

$$
\begin{gathered}
R^{*}{ }_{0 \nu}=\zeta_{0} j_{5}^{*} g_{m}{ }^{\rho}\left(S_{m 5 \nu, \rho}-S_{m 5 \rho, \nu}+S_{m n \nu} S_{n 5 \rho}-S_{m n \rho} S_{n 5 \nu}\right), \\
R^{*}{ }_{\nu 0}=0, \\
R^{*}{ }_{00}=A^{2} .
\end{gathered}
$$

In real form

$$
\begin{aligned}
& R_{\mu \nu}=G_{\mu \nu}+T_{\mu \nu ; \rho}^{\rho}-T_{\mu \rho ; \nu}^{\rho}+T^{\rho}{ }_{\nu \nu} T^{\tau}{ }_{\mu \rho}-T^{\rho \rho}{ }_{\tau \rho} T^{\tau}{ }_{\mu \nu} \\
& +T^{\rho}{ }_{0 \nu} T_{\mu \rho}^{0}-T^{\rho \rho}{ }_{0 \rho} T^{0}{ }_{\mu \nu}, \\
& R_{0 \nu}=R^{*}{ }_{0 \nu}=T^{\rho}{ }_{0 \nu ; \rho}-T^{\rho}{ }_{0 \rho ; \nu}+T^{\rho}{ }_{\tau \nu} T^{\tau}{ }_{0 \rho}-T^{\rho}{ }_{\tau \rho} T^{\tau}{ }_{0 \nu} .
\end{aligned}
$$

Hamiltonian spinors. It follows from I. (25) and I. (31) that the quantities

$$
U_{\nu}{ }^{\mu}=\frac{1}{2} j_{5}^{*}\left\langle\Psi / \nu \Gamma_{n} g_{n}{ }^{\mu} \Psi-\Psi \Gamma_{n} g_{n}{ }^{\mu} \Psi_{/ \nu}\right\rangle
$$

and

$$
V_{\nu}{ }^{\mu}=\frac{1}{2} i j_{5}^{*}\left\langle\Psi_{/ \nu} \Gamma_{n} g_{n}{ }^{\mu} \Psi+\Psi \Gamma_{n} g_{n}{ }^{\mu} \Psi_{/ \nu}\right\rangle
$$

are real tensors of gauge index 1 , and

$$
\begin{aligned}
& U_{0}{ }^{0}=\frac{1}{2} \zeta_{0}\left\langle\Psi_{/ 0} \Gamma_{5} \Psi-\Psi \Gamma_{5} \Psi / 0\right\rangle \\
& V_{0}{ }^{0}=\frac{1}{2} i \zeta_{0}\left\langle\Psi_{/ 0} \Gamma_{5} \Psi+\Psi \Gamma_{5} \Psi / 0\right.
\end{aligned}
$$

are real scalars of gauge index 1 . They are invariant under orientations but not under reflection which carries them into their negatives. 
From (71) we have, by I. (28) and I. (30),

$$
\begin{aligned}
\frac{2}{i} j_{5} V_{\nu}{ }^{\mu}=\left\langle\left(\Psi_{, \nu}-i A_{\nu} \Psi-\frac{1}{4} i \Gamma_{m p} S_{m p \nu} \Psi-\frac{1}{2} i \Gamma_{m 5} S_{m 5 \nu} \Psi\right) \Gamma_{n} g_{n}{ }^{\mu} \Psi\right. \\
\left.+\Psi \Gamma_{n} g_{n}{ }^{\mu}\left(\Psi{ }_{, \nu}-i A_{\nu} \Psi-\frac{1}{4} i \Gamma_{m p} S_{m p \nu} \Psi-\frac{1}{2} i \Gamma_{m 5} S_{m 5 \nu} \Psi\right)\right\rangle \\
=g_{n}{ }^{\mu}\left\langle\Psi \Gamma_{n} \Psi\right\rangle_{, \nu}+g_{n}{ }^{\mu}\left\langle\Psi \frac{1}{4} i\left(\Gamma_{m p} \Gamma_{n}-\Gamma_{n} \Gamma_{m p}\right) S_{m p \nu} \Psi\right\rangle \\
+g_{n}{ }^{\mu}\left\langle\Psi \frac{1}{2} i\left(\Gamma_{m 5} \Gamma_{n}-\Gamma_{n} \Gamma_{m 5}\right) S_{m 5 \nu} \Psi\right\rangle \\
=g_{n}{ }^{\mu}\left\langle\Psi \Gamma_{n} \Psi\right\rangle_{, \nu}+g_{n}{ }^{\mu} S_{m n \nu}\left\langle\Psi \Gamma_{m} \Psi\right\rangle-g_{m}{ }^{\mu} S_{m 5 \nu}\left\langle\Psi \Gamma_{5} \Psi\right\rangle .
\end{aligned}
$$

Or, writing

$$
J_{a}=\frac{1}{2} i j_{5}^{*}\left\langle\Psi \Gamma_{a} \Psi\right\rangle
$$

we get

$$
V_{\nu}{ }^{\mu}=g_{n}{ }^{\mu} J_{n / \nu}=g_{n}{ }^{\mu} J_{n, \nu}-g_{n}{ }^{\mu} S_{n m \nu} J_{m}-g_{n}{ }^{\mu} S_{n 5 \nu} J_{5} \text {. }
$$

Finally, if $\Psi$ is homogeneous we get, from (58), (72) and (73),

$$
\begin{gathered}
U_{0}{ }^{0}=i \zeta_{0} A_{0}\left\langle\Psi \Gamma_{5} \Psi\right\rangle-\frac{1}{2} A\langle\Psi \Psi\rangle, \\
V_{0}{ }^{0}=0 .
\end{gathered}
$$

In the next section we shall form the combination

$$
\begin{aligned}
U_{\mu}{ }^{\mu}+V_{\mu}{ }^{\mu}=\frac{1}{2} j_{5}^{*}\left\langle\Psi_{/ \mu} \Gamma_{n} g_{n}{ }^{\mu} \Psi-\Psi \Gamma_{n} g_{n}{ }^{\mu} \Psi_{/ \mu}\right\rangle & \\
& \quad+\frac{1}{2} i j_{5}^{*}\left\langle\Phi_{/ \mu} \Gamma_{n} g_{n}{ }^{\mu} \Phi+\Phi \Gamma_{n} g_{n}{ }^{\mu} \Phi_{/ \mu}\right\rangle
\end{aligned}
$$

from two independent spinor fields $\Psi$ and $\Phi$. The combination, which at first sight seems to be rather unnatural, can be justified by the following considerations.

Let us extend the algebra $\Omega$ to a larger algebra $\Omega_{q}$ generated by the $\Gamma_{a}$ but admitting for coefficients the non-commutative field of real quaternions. These are expressions of the form $a+b i+c j+d k$ where $j i=-i j=k$, $k j=-j k=i, i k=-k i=j$, and $a, b, c, d$ are real numbers. Elements of $\Omega_{q}$ have the form I.(7) where now $\alpha, \beta_{a}, \gamma_{a b}$ are quaternions. One can develop a calculus for spinors over $\Omega_{q}$ (Hamiltonian spinors) in much the same manner as for spinors over $\Omega$, with the injunction that operators are restricted to the subalgebra $\Omega$. Hamiltonian spinors can be written in the form

$$
\Xi=\Phi+j \Psi
$$

where $\Phi$ and $\Psi$ are spinors over $\Omega$. It can be shown that with a suitable definition of the inner product, expression (78) becomes

$$
\frac{1}{2} i j_{5}^{*}\left\langle\Xi_{/ \mu} \Gamma_{n} g_{n}{ }^{\mu} \Xi+\Xi \Gamma_{n} g_{n}{ }^{\mu} \Xi_{/ \mu}\right\rangle \text {. }
$$

Thus, using a suitable algebra, (78) can be regarded as a single entity, constructed from a single Hamiltonian spinor field. As no explicit use will be made of expression (79), the proof is omitted. 


\section{Field Equations.}

7. The Variation Principle. We now come to the concluding step in the construction of spinor geometry and postulate:

(a) There is given a fibre bundle $\Omega(p), \mathfrak{T}(p)$ over the 4-manifold $\mathbb{R}$, characterized by the "frame variables"

$$
A_{0}, \quad A_{\mu}, \quad S_{a b \mu}, \quad A, \quad g_{n \mu} .
$$

(b) There is given a homogeneous Hamiltonian spinor field over $R$, characterized by a pair of homogeneous spinor fields

$$
\phi, \quad \Psi \text {. }
$$

The necessity of postulating a spinor field arises quite apart from formal considerations such as the determination of $j_{0}$. In fact, if there is no spinor field present then it is altogether impossible to "test" the geometrical characteristics of the space, and spinor geometry becomes a meaningless structure. In this respect it differs fundamentally from geometries based on a (metrical or an affine) vector connection in which the same tensors which describe the geometrical characteristics of space can also be used as "test objects" to detect these characteristics. It is largely due to this fact that Riemannian geometry does not require intrinsically any field laws at all. Spinor geometry, on the other hand, is forced to admit field laws if the frame variables and the spinor field are to form a coherent geometrical structure.

Unfortunately there is no compelling mathematical or logical reason for accepting any particular set of field laws, and, apart from certain established principles partly of aesthetical character, we must take hints from physical experience so as to make the geometrical structure resemble the actual physical universe as closely as possible.

Now there are two basic problems to which physical field laws must supply an answer. First, there is the problem of field generation. Given the distribution of matter (mass, charge, spin) one ought to be able to determine, under suitable boundary conditions, the external physical fields generated by it. In the present theory where the distribution of matter is symbolized by a spinor field, the field generation laws must be differential equations in the frame variables of, say, Porsson's type, in which the density function is an expression constructed by means of the spinor field.

The second fundamental problem is this: Given the distribution of the external field, determine the motion of a test particle when brought into the field. More generally, given the distribution of matter and field at a certain instant (the latter in conformity with the field generation equations) determine the rate of change of the matter distribution under the influence of the external field. What one expects to find is a first order linear differential equation for $\phi$ and $\Psi$ of Dirac's type whose coefficients are functions of the frame variables.

In this respect the present theory is in a peculiar situation. There appear to 
be two types of equations of motion-a continuous and a discrete. The continuous equation of motion is the one referred to above; it is derived from a Hamiltonian principle, by an independent variation of the spinor field, and refers to the motion of a $\Psi$-fluid in the external field. On the other hand, it is reasonable to expect that, because of the non-linear character of the field generation equations, the motion of a test particle (i.e. of a point singularity superimposed upon a given external field) is uniquely determined by the field generation equations alone. However, it is not altogether impossible to reconcile the two; for the equation of motion of a test particle can only be obtained by successive approximations, and obviously each step in the approximation will be influenced by the continuous equation of motion which, through the field generation equations, reacts back upon the external field. Since both the field generation equations and the continuous equation of motion are derived from a single variation principle, it can be hoped that they will not contradict each other. In fact, the dual nature of the equation of motion contains the germs of a possibility that quantum laws will ultimately be deducible from this, or perhaps some other similarly constructed, theory.

The following convention will be used for the rest of the paper: If a tensor density is derived from a tensor by multiplying it with $\mathfrak{g}$, it will be denoted by the same letter in Gothic script, and conversely.

If $B_{\mu \nu}$ is a covariant tensor of order 2 then its symmetrical and antisymmetrical parts will be denoted

$$
B_{\underline{\mu \nu}}=\frac{1}{2}\left(B_{\mu \nu}+B_{\nu \mu}\right), \quad B_{\underline{\mu \nu}}=\frac{1}{2}\left(B_{\mu \nu}-B_{\nu \mu}\right) .
$$

A similar notation is used for contravariant tensor densities of order 2.

The field generation equations and the equations of motion are obtained from a single law, condensed in the form of Hamilton's principle. The proposed world Lagrangian density consists of two terms (or term-complexes): the field Lagrangian density $\mathcal{R}_{(f)}$ and the spinor Lagrangian density $\mathcal{R}_{(s)}$, representing the action of "field" and "matter", respectively.

Write

$$
T=\zeta A^{-1}, \quad T>0
$$

where $\zeta$ is +1 or -1 depending on whether $A$ is positive or negative; let, furthermore,

$$
\begin{gathered}
C_{\mu}=T_{, \mu}, \quad C_{0}=0, \\
C_{\mu \nu}=C_{\mu ; \nu}=T_{, \mu, \nu}-\left\{\begin{array}{c}
\rho \\
\mu \nu
\end{array}\right\} T_{, \rho}, \\
C_{\mu 0}=C_{0 \mu}=C_{00}=0, \\
D_{\mu \nu \rho \sigma}=C_{\mu \nu} C_{\rho \sigma}, \quad D_{\nu \rho}=g^{\mu \sigma} D_{\mu \nu \rho \sigma} .
\end{gathered}
$$

$C_{\mu}$ is a curl-free vector of gauge index $-1, C_{\mu \nu}$ is a symmetric tensor of gauge 
index $-1 . D_{\mu \nu \rho \sigma}$ is of gauge index -2 and $D_{\nu \rho}$ is gauge invariant; they have the symmetries

$$
D_{\mu \nu \rho \sigma}=D_{\nu \mu \rho \sigma}=D_{\mu \nu \sigma \rho}=D_{\rho \sigma \mu \nu}, \quad D_{\nu \rho}=D_{\rho \nu} .
$$

With these notations the field Lagrangian is postulated to have the form

$$
\mathfrak{Z}_{(f)}=\left(\frac{1}{2} \mathfrak{F}^{\alpha \beta}+\gamma \mathfrak{g}^{\alpha \beta}\right)\left(R_{\alpha \beta}^{*}+\frac{1}{2} \Lambda D_{\alpha \beta}\right)
$$

where, for the purposes of this definition, we let the suffixes $\alpha, \beta$ go through the values $0,1, \cdots, 4 . \gamma$ and $\Lambda$ are universal constants. Since $\widetilde{F}^{\mu \nu}, R^{*}{ }_{\mu \nu}$ and $D_{\mu \nu}$ are gauge invariant and $\mathrm{g}^{\mu \nu}$ is of gauge index $-2, \gamma$ must be of gauge index 2 and $\Lambda$ is an absolute constant. $\Omega_{(f)}$ itself is an absolute (gauge invariant) world density. Since $\gamma$ is of gauge index 2 , the equation $|\gamma|=1$ determines an absolute unit of length; presumably it is of the same order of magnitude as the classical radius (or Compton wave-length) of the electron.

Of the two factors in expression (6) the first one is a familiar combination; it unites $F_{\mu \nu}$ and $g_{\mu \nu}$ (representing the electromagnetic and gravitational fields, respectively) into a single tensor, an idea which is familiar from affine theories with a non-symmetrical metric tensor. The combination $R^{*}{ }_{\alpha \beta}+\Lambda D_{\alpha \beta}$ is a natural counterpart of the former, in view of the symmetries (5) of $D_{\mu \nu \rho \sigma}$ which contrast with the symmetries II. (28) and II. (37) of $R_{\nu \mu \rho \sigma}$ and $G_{\mu \nu \rho \sigma}$. The combination has recently been investigated by the author ${ }^{20}$ and shown to lead to a reasonable theory of general relativity if $T$ is interpreted as "cosmic time".

For the spinor Lagrangian density the following expression suggests itself:

$$
\mathfrak{Z}_{(s)}=\kappa\left(\mathfrak{U}_{\alpha}{ }^{\alpha}+\mathfrak{B}_{\alpha}{ }^{\alpha}\right)
$$

where $U_{\nu}{ }^{\mu}, V_{\nu}{ }^{\mu}, U_{0}{ }^{0}, V_{0}^{0}$ are the tensors defined in II. (70)-(73) and $\kappa$ is a universal constant of gauge index 3 . Although $\kappa$ is gauge dependent, it cannot be used to introduce a second absolute unit of length, since its value also depends on the normalization of $\Psi$ and $\Phi$. On the contrary, it can be used to normalize $\Psi$ and $\Phi$ by assigning a convenient value to $\kappa$. Under the influence of a reflection, $\kappa$ changes into $-\kappa$.

Expression (7) is a natural corollary of the Lagrangian of quantum field theories, except for the unorthodox term $\mathfrak{B}_{\alpha}{ }^{\alpha}$. The necessity for introducing this term will be apparent later.

Using the explicit expressions (1)-(4) and formulæ (8), (23), (29), (60)(67) and (75)-(78) of part II, we get for $\Omega_{(f)}$ and $\ell_{(s)}$

$$
\begin{aligned}
\mathfrak{l}_{(f)}= & \mathfrak{g} g_{m}{ }^{\sigma}\left(\frac{1}{2} g^{\nu \rho} g_{n}{ }^{\mu}\left(A_{\mu, \nu}-A_{\nu, \mu}\right)+\gamma g_{n}{ }^{\rho}\right)\left(S_{m n \rho, \sigma}-S_{m n \sigma, \rho}\right. \\
& \left.+S_{m k \rho} S_{k n \sigma}-S_{m k \sigma} S_{k n \rho}+S_{m 5 \rho} S_{5 n \sigma}-S_{m 5 \sigma} S_{5 n \rho}\right) \\
& +\frac{1}{2} \zeta_{0} j_{5} A_{0, \nu} \mathfrak{g} g^{\mu \nu} g_{m}{ }^{\rho}\left(S_{m 5 \mu, \rho}-S_{m 5 \rho, \mu}+S_{m n \mu} S_{n 5 \rho}-S_{m n \rho} S_{n 5 \mu}\right) \\
& +\frac{1}{2} \gamma \Lambda \mathfrak{g} g^{\mu \sigma} g^{\nu \rho}\left(T_{, \mu, \nu}-\left\{\begin{array}{c}
\lambda \\
\mu \nu
\end{array}\right\} T_{, \lambda}\right)\left(T_{, \rho, \sigma}-\left\{\begin{array}{c}
\tau \\
\mu \nu
\end{array}\right\} T_{, \tau}\right)+2 \gamma \epsilon_{5} \mathfrak{g} A^{2},
\end{aligned}
$$

${ }^{20}$ Reference [11], also [12]. 


$$
\begin{aligned}
\mathfrak{R}_{(s)}=\frac{1}{2} \kappa j_{5} \mathfrak{g} \mathfrak{G}\left\langle\Psi{ }_{\mu} \Gamma_{n} g_{n}{ }^{\mu} \Psi\right. & \left.-\Psi \Gamma_{n} g_{n}{ }^{\mu} \Psi_{/ \mu}\right\rangle \\
& +i \zeta_{0} \kappa \mathfrak{g} A_{0}\left\langle\Psi \Gamma_{5} \Psi\right\rangle-\frac{1}{2} \kappa \mathfrak{g} A\langle\Psi \Psi\rangle \\
& \quad+\kappa \mathfrak{g} g_{m}{ }^{\mu}\left(J_{m, \mu}-S_{m n \mu} J_{n}-S_{m 5 \mu} J_{5}\right) .
\end{aligned}
$$

Hamilton's principle requires that

$$
\delta \int\left(R_{(f)}+R_{(s)}\right) d^{4} x=0
$$

for any variations of the field variables which vanishes at the boundary of the domain of integration. Here the independent field variables are the frame variables

$$
A_{0}, A_{\mu}, S_{m n \mu}, S_{m 5 \mu}, A, g_{n}{ }^{\mu}
$$

and the spinor field variables

$$
J_{m}, J_{5}, \Psi .
$$

The fundamental weakness of the variation principle (10) as compared with the rest of the postulates is that it is essentially a physical law whose justification seems to rest almost wholly on empirical considerations. What is missing is some geometrical principle which would lead uniquely to (6) and (7) or perhap to some other similarly constructed expression. At present the only justification on geometrical grounds appears to be the formally very satisfactory structure of the field laws. The next two sections are devoted to the derivation of these field laws.

Remark. We may assume without loss of generality that $\gamma>0, \kappa>0$ and $\zeta=1$ in (1) so that $A>0$ everywhere. Suppose that $\gamma$ is negative. Then reversing the sign of $A_{0}, A_{\mu}$ and the Lagrangian and taking the conjugate imaginary of the Minkowski and spinor representations in which every vector, operator and spinor is replaced by its conjugate imaginary as defined in I. (9), nothing will change in the Lagrangian except the sign of $\gamma$ and possibly the sign of $\kappa$. In fact, writing

$$
\begin{array}{lll}
\tilde{A}_{0}=-A_{0}, & \tilde{A}_{\mu}=-A_{\mu}, \quad \widetilde{S}_{a b \mu}=\left(S_{a b \mu}\right)^{*}, \quad \tilde{g}_{n \mu}=\left(g_{n \mu}\right)^{*}, \\
\tilde{J}_{a}=\epsilon_{5}\left(J_{a}\right)^{*}, & \tilde{\zeta}_{0}=\epsilon_{5} \zeta_{0},
\end{array}
$$

we find from I. (10) and II. (14) that

$$
\left(\Psi_{/ \mu}\right)^{-}=\Psi^{-}{ }_{, \mu}-\left(i \widetilde{A}_{\mu}+\frac{1}{4} i \Gamma_{a b} \widetilde{S}_{a b \mu}\right) \Psi^{-},
$$

which is the spin derivative of $\Psi^{-}$with respect to the new components of connection. Therefore, by I. (19), the value of the first, second and last term in (9) will receive a factor $\epsilon_{5}$, and also the third term since the phase factor of $\Psi^{-}$ in II. (54) is $\exp \left\{\frac{1}{2} \Gamma_{5} \epsilon_{5} \tilde{\zeta}_{0} A x_{0}\right\}$. Expression (8) will, of course, remain unchanged except the terms containing $A_{0}$ and $A_{\mu}$ which reverse their signs. 
The sign of $\kappa$ (in case it is negative) can be changed by a reflection of the spinor frame. Finally, if $A<0$ throughout $R$, we replace $A, \zeta_{0}, J_{5}, S_{m 5 \mu}$ by their negatives and $\Psi$ by $\Gamma_{5} \Psi$; again the Lagrangian remains unaltered by these operations. All these transformations lead to equivalent descriptions of the same geometrical situation; the assumptions $\gamma>0, \kappa>0$ fix the chirality of the spinor and Minkowski representations with respect to the base frame and the assumption $A>0$ fixes the sense of time flow.

8. Variation of the Frame Variables. In order to perform the variation of $\mathfrak{R}_{(f)}$, write

$$
\begin{aligned}
\delta \int \mathfrak{R}_{(f)} d^{4} x=\int\left\{\mathfrak{R}^{0} \delta A_{0}+\mathfrak{R}^{\mu} \delta A_{\mu}\right. & +\mathfrak{R}^{\mu}{ }_{m n} \delta S_{m n \mu}+{\mathfrak{\mathfrak { R } ^ { \mu }}}_{m 5} \delta S_{m 5 \mu} \\
& \left.+\mathfrak{\Omega} \delta A+\mathfrak{S}_{m \mu} \delta g_{m}{ }^{\mu}\right\} d^{4} x
\end{aligned}
$$

where $\mathfrak{R}_{n m}^{\mu}=-\mathfrak{R}^{\mu}{ }_{m n}$ and the integration is extended over some finite region of $\mathbb{Q}$ covered by a single $\mathcal{X}$-frame. Assuming that the variation of the field quanities vanishes at the boundary of the region, we obtain by routine calculation

$$
\begin{aligned}
& \mathfrak{R}^{0}=-\frac{1}{2} \Re^{0 \nu}, \\
& \mathfrak{2}^{\mu}=-\Re^{\mu \nu}, \nu, \\
& L_{\lambda \mu \nu}=\frac{1}{4} F_{\mu \nu ; \lambda}-\frac{1}{4}\left(g_{\lambda \mu} F_{\nu ; \rho}^{\rho}-g_{\lambda \nu} F^{\rho}{ }_{\mu ; \rho}\right)+\frac{1}{4} F_{\lambda}^{\rho}\left(T_{\rho \mu \nu}-T_{\rho \nu \mu}\right) \\
& -\frac{1}{4}\left(T_{\lambda \mu \rho} F^{\rho}{ }_{\nu}-T_{\lambda \nu \rho} F_{\mu}^{\rho}\right)-\frac{1}{4}\left(g_{\lambda \mu} T_{\nu \rho \sigma}-g_{\lambda \nu} T_{\mu \rho \sigma}\right) F^{\rho \sigma} \\
& -\frac{1}{4}\left(F_{\lambda \mu} T_{\nu \rho}^{\rho}-F_{\lambda \nu} T_{\mu \rho}^{\rho}\right)+\frac{1}{4} F_{\lambda}^{0}\left(T_{0 \mu \nu}-T_{0 \nu \mu}\right) \\
& +\frac{1}{4}\left(g_{\lambda \mu} T_{0 \nu \sigma}-g_{\lambda \nu} T_{0 \mu \sigma}\right) F^{0 \sigma} \\
& +\gamma\left(T_{\lambda \mu \nu}-T_{\lambda \nu \mu}\right)+\gamma\left(g_{\lambda \mu} T^{\rho}{ }_{\nu \rho}-g_{\lambda \nu} T^{\rho}{ }_{\mu \rho}\right) \text {, } \\
& L_{\lambda \mu 0}=-\frac{1}{2} g_{\lambda \mu} T_{0 \rho \sigma} F^{\rho \sigma}-\frac{1}{2} T_{0 \lambda \rho} F^{\rho}{ }_{\mu}+\frac{1}{2} F^{\rho}{ }_{\lambda} T_{0 \rho \mu}-\frac{1}{2} F_{\lambda \mu} T^{\rho}{ }_{0 \rho} \\
& -\frac{1}{2} F_{0 \lambda ; \mu}-\frac{1}{2} F_{0 ; \rho}^{\rho} g_{\lambda \mu}-\frac{1}{2} T_{\lambda \mu \rho} F_{0}^{\rho}-\frac{1}{2} F_{0 \lambda} T_{\mu \rho}^{\rho} \\
& +2 \gamma T_{0 \lambda \mu}+2 \gamma g_{\lambda \mu} T^{\rho}{ }_{0 \rho} \text {, } \\
& L=-\gamma \Lambda A^{-2} C_{; \mu ; \nu}^{\mu \nu}+4 \gamma \epsilon_{5} A, \\
& H_{\mu \nu}=\frac{1}{2} F^{\rho \sigma} R_{\mu \rho \sigma \nu}-\frac{1}{2} F^{\rho}{ }_{\nu} R_{\mu \rho}+\frac{1}{2} F^{\rho}{ }_{\nu} R_{\rho \mu}+\frac{1}{2} F^{\rho}{ }_{\mu} R_{\rho \nu} \\
& +\frac{1}{2} F^{0 \rho} R_{\mu 0 \rho \nu}+\frac{1}{2} F^{0}{ }_{\nu} R_{0 \mu}+\frac{1}{2} F^{0}{ }_{\mu} R_{0 \nu} \\
& +2 \gamma R_{\mu \nu}+\gamma \Lambda K_{\mu \nu}-g_{\mu \nu} L_{(f)} \text {, }
\end{aligned}
$$

where

$$
K_{\mu \nu}=-C_{\mu} C_{\nu ; \rho}^{\rho}-C_{\nu} C_{\mu ; \rho}^{\rho}+C_{\mu \nu} C_{\rho}^{\rho}+C^{\rho} C_{\mu \nu ; \rho}
$$


The calculation is indicated below.

(14) and (15): The term containing $A_{\mu}$ in (8) has the form $A_{\mu, \nu} \Re^{\mu \nu}$, and

$$
\int \delta A_{\mu, \nu} \Re^{\mu \nu} d^{4} x=-\int \Re^{\mu \nu}{ }_{, \nu} \delta A_{\mu} d^{4} x
$$

since $\delta A_{\mu}=0$ on the boundary. The same proof applies to the variation of $A_{0}$.

(16): Write the part of $\&_{(f)}$ which involves $S_{m n \nu}$ in the form

$$
\begin{aligned}
\mathfrak{g}\left(-\frac{1}{2} g_{m}{ }^{\mu} F_{n}^{\nu}+\gamma g_{m}{ }^{\mu} g_{n}{ }^{\nu}\right)\left(S_{m n \nu, \mu}-\right. & \left.S_{m n \nu, \mu}+S_{m k \nu} S_{k n \mu}-S_{m k \mu} S_{k n \nu}\right) \\
& +\frac{1}{2} \zeta_{0} j_{5}\left(g_{m}{ }^{\tau} \mathfrak{F}_{0}^{\rho}-g_{m}{ }^{\rho} \mathfrak{F}_{0}^{\tau}\right) S_{m n \tau} S_{n 5 p}
\end{aligned}
$$

and replace every $S_{m n \nu}$ therein by $\frac{1}{2}\left(S_{m n \nu}-S_{n m \nu}\right)$.

Using partial integration to get rid of the derivatives, we obtain

$$
\begin{aligned}
\mathfrak{R}^{\tau}{ }_{m n}=\frac{1}{4}\left(g_{m}{ }^{\rho} \mathfrak{F}_{n}^{\tau}-\right. & \left.g_{n}{ }^{\rho} \mathfrak{F}_{m}^{\tau}-g_{m}{ }^{\tau} \mathfrak{F}_{n}^{\rho}+g_{n}{ }^{\tau} \mathfrak{F}_{m}^{\rho}\right)_{, \rho} \\
& -\frac{1}{4}\left(g_{m}{ }^{\rho} \mathfrak{F}_{k}^{\tau}{ }_{k} S_{n k \rho}-g_{n}{ }^{\rho} \mathfrak{F}^{\tau}{ }_{k} S_{m k \rho}+g_{k}{ }^{\tau} \mathfrak{F}_{n}^{\rho} S_{k m \rho}-g_{k}{ }^{\tau} \mathfrak{F}_{m}^{\rho}{ }_{m} S_{k n \rho}\right. \\
& \left.-g_{m}{ }^{\tau} \mathfrak{F}_{k}^{\rho}{ }_{k} S_{n k \rho}+g_{n}{ }^{\tau} \mathfrak{F}_{k}^{\rho}{ }_{k} S_{m k \rho}-g_{k}{ }^{\rho} \mathfrak{F}_{n}{ }_{n} S_{k m \rho}+g_{k}{ }^{\rho} \mathfrak{F}_{m}^{\tau}{ }_{m} S_{k n \rho}\right) \\
& -\gamma\left(\mathfrak{g}_{n}{ }^{\tau} g_{m}{ }^{\rho}-g_{m}{ }^{\tau} \mathfrak{g}_{n}{ }^{\rho}\right)_{, \rho} \\
& +\gamma\left(\mathfrak{g}_{k}{ }^{\tau} g_{m}{ }^{\rho} S_{n k \rho}-\mathfrak{g}_{k}{ }^{\tau} g_{n}{ }^{\rho} S_{m k \rho}-\mathfrak{g}_{k}{ }^{\rho} g_{m}{ }^{\tau} S_{n k \rho}+\mathfrak{g}_{k}{ }^{\rho} g_{n}{ }^{\tau} S_{m k \rho}\right) \\
& +\frac{1}{4} \zeta_{0} j_{5}{ }^{*}\left(\mathfrak{F}^{0 \tau} g_{m}{ }^{\rho} S_{n 5 \rho}-\mathfrak{F}^{0 \tau} g_{n}{ }^{\rho} S_{m 5 \rho}-\mathfrak{F}^{0 \rho} g_{m}{ }^{\tau} S_{n 5 \rho}+\mathfrak{F}^{0 \rho} g_{n}{ }^{\tau} S_{m 5 \rho}\right) .
\end{aligned}
$$

To prove (16) it is sufficient to remark that the expression, when multiplied by $g_{m \mu} g_{n v} g_{\lambda \tau}$, becomes identical with expression (16) in a geodesic $\mathcal{X}$-frame and geodesic orientation.

(17): The part of $\mathfrak{R}_{(f)}$ which involves $S_{m 5 \nu}$ can be put in the form

$$
\begin{aligned}
-\frac{1}{2} \mathfrak{g}\left(g_{m}{ }^{\tau} F^{\rho}{ }_{n}-g_{n}{ }^{\tau} F^{\rho}{ }_{m}\right) S_{m 5 \tau} S_{n 5 \rho}+ & \gamma \mathfrak{g}\left({g_{m}}^{\tau}{g_{n}}^{\rho}-{g_{n}}^{\tau} g_{m}{ }^{\rho}\right) S_{m 5 \tau} S_{n 5 \rho} \\
& +\frac{1}{2} \zeta_{0} j_{5}{ }^{*}\left(\mathfrak{F}^{0 \tau} g_{m}{ }^{\rho}-\mathfrak{F}^{0 \rho} g_{m}{ }^{\tau}\right)\left(S_{m 5 \tau, \rho}-S_{m n \rho} S_{n 5 \tau}\right)
\end{aligned}
$$

which gives

$$
\begin{aligned}
{\mathfrak{R}^{\tau}}_{m 5}=-\frac{1}{2} \mathfrak{g}\left(g_{m}{ }^{\tau}{F^{\rho}}_{n}\right. & \left.-g_{n}{ }^{\tau}{F^{\rho}}_{m}+g_{n}{ }^{\rho}{F^{\tau}}_{m}-g_{m}{ }^{\rho}{F^{\tau}}_{n}\right) S_{n 5 \rho} \\
& +2 \gamma \mathfrak{g}\left(g_{m}{ }^{\tau} g_{n}{ }^{\rho}-g_{n}{ }^{\tau} g_{m}{ }^{\rho}\right) S_{n 5 \rho} \\
& -\frac{1}{2} \zeta_{0} j_{5}{ }^{*}\left(\mathfrak{F}^{0 \tau} g_{m}{ }^{\rho}-\mathfrak{F}^{0 \rho} g_{m}{ }^{\tau}\right)_{, \rho}+\frac{1}{2} \zeta_{0} j_{5}{ }^{*}\left(\mathfrak{F}^{0 \tau} g_{n}{ }^{\rho}-\mathfrak{F}^{0 \rho} g_{n}{ }^{\tau}\right) S_{m n \rho} .
\end{aligned}
$$

From this (17) follows as in the previous case.

(18): The terms containing $A=T^{-1}$ are

$$
\frac{1}{2} \gamma \Lambda \mathfrak{g} g^{\mu \sigma} g^{\nu \rho} T_{, \mu ; \nu} T_{, \rho ; \sigma}+2 \gamma \epsilon_{5} \mathfrak{g} A^{2}
$$

from which the result is obtained through repeated covariant partial integration of the first term and the observation that $\delta T=-A^{-2} \delta A$. 
(19): This can be proved by writing $\mathfrak{R}_{(f)}$ in the form

$$
\begin{aligned}
\mathfrak{R}_{(f)}=\frac{1}{2} F_{\mu \nu} \mathfrak{g} g_{m}{ }^{\sigma} g_{n}{ }^{\mu} g^{\nu \rho} R_{m n \rho \sigma}+\frac{1}{2} \zeta_{0} j_{5} F_{0 \nu} \mathfrak{g} g_{m}{ }^{\rho} g^{\mu \nu} R_{m 5 \mu \rho} & \\
& +\gamma \mathfrak{g} g_{m}{ }^{\sigma} g_{n}{ }^{\rho} R_{m n \rho \sigma}+2 \gamma \epsilon_{5} \mathfrak{g} A^{2} \\
& +\frac{1}{2} \gamma \Lambda \mathfrak{g}^{\mu \sigma} g^{\nu \rho}\left(C_{\mu, \nu}+\left\{\begin{array}{c}
\lambda \\
\mu \nu
\end{array}\right\} C_{\lambda}\right)\left(C_{\rho, \sigma}-\left\{\begin{array}{c}
\tau \\
\rho \sigma
\end{array}\right\} C_{\tau}\right)
\end{aligned}
$$

and using the relations

$$
\begin{aligned}
\delta \mathrm{g} & =-\mathfrak{g} g_{m \nu} \delta g_{m}{ }^{\nu} \quad \text { (no summation!), } \\
\delta g^{\mu \nu} & =g_{m}{ }^{\mu} \delta g_{m}{ }^{\nu}+g_{m}{ }^{\nu} \delta g_{m}{ }^{\mu}, \quad \delta g_{\rho \sigma}=-g_{\rho \mu} g_{\sigma \nu} \delta g^{\mu \nu} .
\end{aligned}
$$

The tensors (14)-(19) are functions of $1+4+24+16+1+10=56$ independent tensor components, viz. $A_{0}, A_{\mu}, T_{\mu \nu \lambda}, T_{\mu 0 \lambda}, A, g_{\mu \nu}$. Now the total number of distinct components of the tensors $L^{0}, L^{\mu}, L_{\lambda_{\mu \nu}}, L_{\lambda_{\mu} 0}, L, H_{\mu \nu}$ is $1+$ $4+24+16+1+16=62$, which implies that there exist six identical relations between them. To obtain these identities, let us perform a variation of $\int \mathfrak{R}_{(f)} d^{4} x$ by applying a reorientation

$$
\exp \{\boldsymbol{\epsilon} \mathbf{P}(x)\}=\mathbf{I}+\epsilon \mathbf{P}+\frac{1}{2} \epsilon^{2} \mathbf{P}^{2}+\cdots
$$

to the Minkowski frames such that $\mathbf{P}=0$ at the boundary of the domain of integration. $\epsilon$ is a positive number which we let tend to zero. Then, apart from terms of order $\epsilon^{2}$,

$$
\begin{aligned}
\delta S_{m n \rho} & =\epsilon\left(P_{m k} S_{k n \rho}+P_{n k} S_{m k \rho}+P_{m n, \rho}\right), \\
\delta S_{m 5 \rho} & =\epsilon P_{m n} S_{n 5 \rho} \\
\delta g_{m}{ }^{\rho} & =\epsilon P_{m n} g_{n}{ }^{\rho}
\end{aligned}
$$

by formulas II. (19) and II. (20), hence

$$
\begin{aligned}
& \delta \int \mathfrak{R}_{(f)} d^{4} x=-\epsilon \int\left\{\mathfrak{S}_{m \rho} g_{n}{ }^{\rho} P_{m n}+\mathfrak{R}_{m k}^{\rho} S_{n k \rho} P_{m n}+\mathfrak{R}^{\rho}{ }_{k n} S_{m k \rho} P_{m n}\right. \\
& \left.+\mathfrak{R}_{m n}^{\rho} P_{m n, \rho}+{\mathfrak{\mathfrak { R } ^ { \rho }}}_{m 5} S_{n 5 \rho} P_{m n}\right\} d^{4} x \\
& =-\frac{1}{2} \epsilon \int P_{m n}\left\{\mathfrak{S}_{m \rho} g_{n}{ }^{\rho}-\mathfrak{S}_{n \rho} g_{m}{ }^{\rho}+\mathfrak{R}^{\rho}{ }_{m k} S_{n k \rho}-\mathfrak{R}_{n k}^{\rho} S_{m k \rho}\right. \\
& +\mathfrak{R}^{\rho}{ }_{k n} S_{m k \rho}-\mathfrak{R}^{\rho}{ }_{k m} S_{n k \rho}-\mathfrak{R}^{\rho}{ }_{m n, \rho}+\mathfrak{R}^{\rho}{ }_{n m, \rho} \\
& \left.+\mathfrak{R}^{\rho}{ }_{m 5} S_{n 5 \rho}-\mathfrak{R}^{\rho}{ }_{n 5} S_{m 5 \rho}\right\} d^{4} x \text {. }
\end{aligned}
$$

Now $\mathfrak{R}_{(f)}$ is independent of reorientations and $P_{m n}=-P_{n m}$ is arbitrary, therefore the expression in the curled brackets must vanish. Multiplying by $h_{m \mu} h_{n \nu}$ and using a geodesic frame we obtain

$$
H_{\mu \nu}=L_{\mu \nu ; \rho}^{\rho}+L_{\mu \sigma}^{\rho} T_{\nu \rho}^{\sigma}-L_{\nu \sigma}^{\rho} T_{\mu \rho}^{\sigma}+\frac{1}{2}\left(L_{\mu 0}^{\rho} T_{\nu \rho}^{0}-L_{\nu 0}^{\rho} T_{\mu \rho}^{0}\right)
$$

which supplies the required identities. 
It is desirable to separate equation (16) into a totally skew symmetric part and a part satisfying the peculiar symmetry conditions below. Write

$$
T_{\mu \nu \lambda}=A_{\mu \nu \lambda}+B_{\mu \nu \lambda}, \quad L_{\lambda \mu \nu}=M_{\lambda \mu \nu}+N_{\lambda \mu \nu}
$$

where

$$
\begin{array}{ll}
A_{\mu \nu \lambda}=\frac{1}{3}\left(T_{\mu \nu \lambda}+T_{\nu \lambda \mu}+T_{\lambda \mu \nu}\right), & M_{\lambda \mu \nu}=\frac{1}{3}\left(L_{\lambda \mu \nu}+L_{\mu \nu \lambda}+L_{\nu \lambda \mu}\right), \\
B_{\mu \nu \lambda}=\frac{1}{3}\left(2 T_{\mu \nu \lambda}+T_{\lambda \nu \mu}+T_{\mu \lambda \nu}\right), & N_{\lambda \mu \nu}=\frac{1}{3}\left(2 L_{\lambda \mu \nu}+L_{\mu \lambda \nu}+L_{\nu \mu \lambda}\right) .
\end{array}
$$

Clearly $A_{\mu \nu \lambda}$ and $M_{\lambda \mu \nu}$ are totally skew symmetric and $B_{\mu \nu \lambda}, N_{\lambda \mu \nu}$ have the following symmetry properties:

$$
\begin{array}{ll}
B_{\mu \nu \lambda}+B_{\nu \mu \lambda}=0, & B_{\mu \nu \lambda}+B_{\nu \lambda \mu}+B_{\lambda \mu \nu}=0, \\
N_{\lambda \mu \nu}+N_{\lambda \nu \mu}=0, & N_{\lambda \mu \nu}+N_{\mu \nu \lambda}+N_{\nu \lambda \mu}=0 .
\end{array}
$$

Also write

$$
\begin{array}{ll}
A_{0 \nu \lambda}=\frac{1}{2}\left(T_{0 \nu \lambda}-T_{0 \lambda \nu}\right), & M_{\lambda \mu 0}=\frac{1}{2}\left(L_{\lambda \mu 0}-L_{\mu \lambda 0}\right), \\
B_{0 \nu \lambda}=\frac{1}{2}\left(T_{0 \nu \lambda}+T_{0 \lambda \nu}\right), & N_{\lambda \mu 0}=\frac{1}{2}\left(L_{\lambda \mu 0}+L_{\mu \lambda 0}\right) .
\end{array}
$$

so that

$$
T_{0 \nu \lambda}=A_{0 \nu \lambda}+B_{0 \nu \lambda}, \quad L_{\lambda \mu 0}=M_{\lambda \mu 0}+N_{\lambda \mu 0} .
$$

From (16), (17), (22)-(28) we obtain, noting that $F_{0 \nu ; \mu}=F_{0 \mu: \nu}$ since $F_{0 \nu}$ is a gradient,

$$
\begin{aligned}
& M_{\lambda \mu \nu}=-\frac{1}{4}\left(F^{\rho}{ }_{\lambda} B_{\mu \nu \rho}+F^{\rho}{ }_{\mu} B_{\nu \lambda \rho}+F^{\rho}{ }_{\nu} B_{\lambda \mu \rho}\right) \\
& -\frac{1}{6}\left(T^{\rho}{ }_{\lambda \rho} F_{\mu \nu}+T^{\rho}{ }_{\mu \rho} F_{\nu \lambda}+T^{\rho}{ }_{\nu \rho} F_{\lambda \mu}\right) \\
& -\frac{1}{4}\left(F_{\lambda}^{0} A_{0 \mu \nu}+F^{0}{ }_{\mu} A_{0 \nu \lambda}+{F^{0}}_{\nu} A_{0 \lambda \mu}\right)+2 \gamma A_{\mu \nu \lambda} \text {, } \\
& N_{\lambda \mu \nu}=\frac{1}{4} F_{\mu \nu ; \lambda}-\frac{1}{4}\left(g_{\lambda \mu} F^{\rho}{ }_{\nu ; \rho}-g_{\lambda \nu} F^{\rho}{ }_{\mu ; \rho}\right)+\frac{1}{2} F_{\lambda}^{\rho} A_{\mu \nu \rho} \\
& -\frac{1}{4}\left(F^{\rho}{ }_{\mu} A_{\nu \lambda \rho}-F^{\rho}{ }_{\nu} A_{\mu \lambda \rho}\right)-\frac{1}{4}\left(g_{\lambda \mu} T_{\nu \rho \sigma}-g_{\lambda \nu} T_{\mu \rho \sigma}\right) F^{\rho \sigma} \\
& +\frac{1}{6} T_{\lambda \rho}^{\rho} F_{\mu \nu}-\frac{1}{12}\left(T_{\mu \rho}^{\rho}{ }_{\mu \nu} F_{\nu \lambda}-T_{\nu \rho}^{\rho}{ }_{\nu \mu} F_{\mu \lambda}\right)+\frac{1}{2} F_{\lambda}^{0} A_{0 \mu \nu} \\
& -\frac{1}{4}\left(F^{0}{ }_{\mu} A_{0 \nu \lambda}-F^{0}{ }_{\nu} A_{0 \mu \lambda}\right)+\frac{1}{4}\left(g_{\lambda \mu} T_{0 \nu \sigma}-g_{\lambda \nu} T_{0 \mu \sigma}\right) F^{0 \sigma} \\
& -\gamma B_{\mu \nu \lambda}+\gamma\left(g_{\lambda \mu} T_{\nu \rho}^{\rho}-g_{\lambda \nu} T^{\rho}{ }_{\mu \rho}\right) \text {, } \\
& M_{\lambda \mu 0}=\frac{1}{2}\left(F_{\lambda}^{\rho} B_{0 \mu \rho}-F^{\rho}{ }_{\mu} B_{0 \lambda \rho}\right)-\frac{1}{2} F_{\lambda \mu} T^{\rho}{ }_{0 \rho}-\frac{1}{2} T_{\lambda \mu \rho} F^{\rho}{ }_{0} \\
& -\frac{1}{4}\left(F_{0 \lambda} T_{\mu \rho}^{\rho}-F_{0 \mu} T_{\lambda \rho}^{\rho}\right)+2 \gamma A_{0 \lambda \mu}, \\
& N_{\lambda \mu 0}=-\frac{1}{2} F_{0 \lambda ; \mu}-\frac{1}{2} F_{0 ; \rho}^{\rho} g_{\lambda \mu}-\frac{1}{2} g_{\lambda \mu} A_{0 \rho \sigma} F^{\rho \sigma}-\frac{1}{2}\left(F_{\lambda}^{\rho} A_{0 \mu \rho}+F^{\rho}{ }_{\mu} A_{0 \lambda \rho}\right) \\
& -\frac{1}{4}\left(F_{0 \lambda} T_{\mu \rho}^{\rho}+F_{0 \mu} T_{\lambda \rho}^{\rho}\right)+2 \gamma B_{0 \lambda \mu}+2 \gamma g_{\lambda \mu} T^{\rho}{ }_{0 \rho} .
\end{aligned}
$$


We perform now the variation of $\Omega_{(s)}$. Using the notation

$$
\Gamma^{\mu}=\mathfrak{g} g_{n}^{\mu} \Gamma_{n}, \quad \Gamma^{0}=\mathfrak{g} \zeta_{0} j_{5} \Gamma_{5}, \quad \Gamma_{\mu}=g_{n \mu} \Gamma_{n}, \quad \Gamma_{0}=\zeta_{0} j_{5} * \Gamma_{5},
$$

we get, from II. (14), II. (70) and the definition of inner product,

$$
\begin{aligned}
& \mathfrak{U}_{\mu}{ }^{\mu}=\frac{1}{2} j_{5} *\left\langle\Psi_{/ \mu} \Gamma^{\mu} \Psi-\Psi \Gamma^{\mu} \Psi_{/ \mu}\right\rangle \\
& =\frac{1}{2} j_{5}{ }^{*} \mathrm{qs}\left\{\left(\Psi^{+}{ }_{, \mu}+\Psi^{+}\left(i A_{\mu}+\frac{1}{4} i \Gamma_{a b} S_{a b \mu}\right)\right) \Gamma^{\mu} \Psi\right. \\
& \left.-\Psi^{+} \Gamma^{\mu}\left(\Psi_{, \mu}-\left(i A_{\mu}+\frac{1}{4} i \Gamma_{a b} S_{a b \mu}\right) \Psi\right)\right\} \\
& =j_{5}{ }^{*} \mathrm{qs}\left\{\frac{1}{2}\left(\Psi^{+}{ }_{, \mu} \Gamma^{\mu} \Psi-\Psi^{+} \Gamma^{\mu} \Psi_{, \mu}\right)+i A_{\mu} \Psi^{+} \Gamma^{\mu} \Psi\right. \\
& +\frac{1}{8} i \Psi^{+} S_{m n \mu}\left(\Gamma_{m n} \Gamma^{\mu}+\Gamma^{\mu} \Gamma_{m n}\right) \Psi \\
& \left.+\frac{1}{4} i \Psi^{+} S_{m 5 \mu}\left(\Gamma_{m 5} \Gamma^{\mu}+\Gamma^{\mu} \Gamma_{m 5}\right) \Psi\right\} \text {. }
\end{aligned}
$$

(9), (10) and (35) give easily

$$
\begin{aligned}
& \mathfrak{R}^{0}=-i j_{5}{ }_{\kappa}\left\langle\Psi \Gamma^{0} \Psi\right\rangle \\
& \mathfrak{R}^{\mu}=-i j_{5}{ }^{*}\left\langle\Psi \Gamma^{\mu} \Psi\right\rangle \\
& M_{\lambda \mu \nu}=-\frac{1}{8} j_{5} *_{\kappa}\left\langle\Psi\left(\Gamma_{\lambda} \Gamma_{\mu} \Gamma_{\nu}-\Gamma_{\nu} \Gamma_{\mu} \Gamma_{\lambda}\right) \Psi\right\rangle \\
& N_{\lambda \mu \nu}=\frac{1}{2} \kappa\left(g_{\lambda \mu} J_{\nu}-g_{\lambda \nu} J_{\mu}\right) \\
& M_{\lambda \mu 0}=-\frac{1}{4} j_{5}{ } \kappa\left\langle\Psi\left(\Gamma_{\lambda} \Gamma_{\mu} \Gamma_{0}-\Gamma_{0} \Gamma_{\mu} \Gamma_{\lambda}\right) \Psi\right\rangle \\
& N_{\lambda \mu 0}=\kappa g_{\lambda \mu} J_{0} \\
& L=\frac{1}{2} \kappa\langle\Psi \Psi\rangle \\
& H_{\mu \nu}=-\frac{1}{2} j_{5}{ }^{*} \kappa\langle\Psi / \nu \\
&
\end{aligned}
$$

where the tensors on the left are the ones determined from (13) and given by (14), (15), (30), (31), (32), (33), (18) and (19), respectively.

From formulæ (39) and (41) we can now see quite clearly why it was necessary to introduce the $\mathfrak{B}_{\mu}{ }^{\mu}$ term into the Lagrangian. Without this term the right hand sides of (39) and (41) would be identically zero, which would impose an artificial and wholly unwarranted symmetry condition upon the tensors $L_{\lambda \mu \nu}$ and $L_{\lambda \mu 0}$.

From (39) we obtain $-N^{\nu}{ }_{\mu \nu}=\frac{3}{2}{ }_{\kappa} J_{\mu}$, and from (31)

$$
-N^{\nu}{ }_{\mu \nu}=-\frac{1}{2} F_{\mu ; \rho}^{\rho}-\frac{3}{4} B_{\mu \rho \sigma} F^{\rho \sigma}+\frac{3}{4} B_{0 \mu \rho} F^{0 \rho}+\frac{1}{4} F_{\mu}^{\nu} B_{\nu \rho}^{\rho}+2 \gamma B^{\rho}{ }_{\mu \rho}
$$

hence

$$
\kappa J_{\mu}=-\frac{1}{3} F_{\mu ; \rho}^{\rho}-\frac{1}{2} B_{\mu \rho \sigma} F^{\rho \sigma}+\frac{1}{2} B_{0 \mu \rho} F^{0 \rho}+\frac{1}{6} F^{\nu}{ }_{\mu} B^{\rho}{ }_{\nu \rho}+\frac{4}{3} \gamma B^{\rho}{ }_{\mu \rho} .
$$

Similarly from (33) and (41) we get

$$
\kappa J_{0}=-\frac{3}{8} F_{0 ; \rho}^{\rho}-\frac{1}{4} A_{0 \rho \sigma} F^{\rho \sigma}+\frac{1}{8} F^{\lambda}{ }_{0} T^{\rho}{ }_{\lambda \rho}+\frac{3}{2} \gamma B^{\rho}{ }_{0 \rho} .
$$


9. The Field Equations. Before giving the field equations their final form, let us write down the equations of motion. These are obtained from the Hamiltonian principle and (9) through variation of $J_{n}, J_{5}$ and $\Psi$ while the frame variables are being kept fixed.

Variation of $J_{n}$ gives

$$
\left(\mathfrak{g} g_{n}{ }^{\mu}\right)_{, \mu}+\mathfrak{g} g_{m}{ }^{\mu} S_{m n \mu}=0
$$

from which we find, by using a geodesic frame and orientation,

$$
T_{\nu \mu}^{\mu}=B^{\mu \mu}=0 \text {. }
$$

Variation of $J_{5}$ gives

$$
T^{\mu}{ }_{0 \mu}=B_{0 \mu}^{\mu}=0 .
$$

Finally, variation of $j_{5}{ }^{*} \Psi^{+}$gives, by (35), noting that $q s\left\{\left(\delta \Psi^{+}\right) \Phi\right\}=0$ for every $\delta \Psi^{+}$implies $\Phi=0$,

$$
\begin{aligned}
& -\Gamma^{\mu} \Psi_{, \mu}-\frac{1}{2} \Gamma_{, \mu}^{\mu} \Psi+i A_{\mu} \Gamma^{\mu} \Psi+\frac{1}{8} i S_{m n \mu}\left(\Gamma_{m n} \Gamma^{\mu}+\Gamma^{\mu} \Gamma_{m n}\right) \Psi \\
& +\frac{1}{4} i S_{m 5 \mu}\left(\Gamma_{m 5} \Gamma^{\mu}+\Gamma^{\mu} \Gamma_{m 5}\right) \Psi+i A_{0} \Gamma^{0} \Psi-\frac{1}{2} A j_{5} g \Psi=0
\end{aligned}
$$

Here

$$
\begin{aligned}
S_{m n \mu} \Gamma_{m n} \Gamma^{\mu}=\mathfrak{g} S_{m n \mu} \Gamma_{m n} \Gamma_{k} g_{k}{ }^{\mu} & \\
& =\mathfrak{g} S_{m n \mu} \Gamma_{k} g_{k}{ }^{\mu} \Gamma_{m n}+\frac{2}{i} \mathfrak{g} S_{m n \mu} \Gamma_{m} g_{n}{ }^{\mu}-\frac{2}{i} \mathfrak{g} S_{m n \mu} \Gamma_{n} g_{m}{ }^{\mu} \\
& =S_{m n \mu} \Gamma^{\mu} \Gamma_{m n}+\frac{4}{i} \Gamma^{\mu}{ }_{, \mu}
\end{aligned}
$$

by (46), and

$$
\begin{aligned}
S_{m 5 \mu} \Gamma_{m 5} \Gamma^{\mu} & =\mathfrak{g} S_{m 5 \mu} \Gamma_{m 5} \Gamma_{k} g_{k}{ }^{\mu} \\
& =\mathfrak{g} S_{m 5 \mu} \Gamma_{k} g_{k}{ }^{\mu} \Gamma_{m 5}-\frac{2}{i} \mathfrak{g} S_{m 5 \mu} \Gamma_{5} g_{m}{ }^{\mu} \\
& =S_{m 5 \mu} \Gamma^{\mu} \Gamma_{m 5}
\end{aligned}
$$

by (48). Hence (49), (50) and (51) give

$$
\begin{aligned}
-\Gamma^{\mu} \Psi_{, \mu}+i A_{\mu} \Gamma^{\mu} \Psi+\frac{1}{4} i S_{m n \mu} \Gamma^{\mu} \Gamma_{m n} \Psi+\frac{1}{2} i S_{m 5 \mu} \Gamma^{\mu} \Gamma_{m 5} \Psi \\
+i A_{0} \Gamma^{0} \Psi-\frac{1}{2} A j_{5} \mathfrak{g} \Psi=0 .
\end{aligned}
$$

This is the equation of motion; in symbolic notation

$$
\Gamma^{\mu} \Psi_{/ \mu}+\Gamma^{0} \Psi_{/ 0}=0 .
$$

Note that the equation of motion obtains this simple form only in consequence of the auxiliary equations (47) and (48) which, on the other hand, were obtained through the variation of $J_{a}$. 
Variation of $\Psi$ gives the adjoint equation

$$
\Psi^{+}{ }_{\mu} \Gamma^{\mu}+\Psi^{+}{ }_{10} \Gamma^{0}=0
$$

which, of course, is equivalent to (53). From (52) and (53) we obtain, multiplying the first one by $\Psi^{+}$from the left, the second one by $\Psi$ from the right, forming the quarterspur and subtracting,

$$
\mathfrak{U}_{\mu}{ }^{\mu}+i j_{5} * A_{0}\left\langle\Psi \Gamma^{0} \Psi\right\rangle-\frac{1}{2} A \mathfrak{g}\langle\Psi \Psi\rangle=0
$$

which with (9) and the auxiliary equations (47), (48) gives

$$
\mathfrak{R}_{(s)}=\kappa \Im_{, \mu}^{\mu} .
$$

By a somewhat lengthy calculation which will not be reproduced here, it can be shown that the relations (21) also hold for the quantities defined by the expressions (38)-(41), (43). They hold as a consequence of the equations of motion, but independently of the field equations. The proof can be carried out similarly to the proof of (21), by performing a variation of $\int \mathfrak{I}_{(s)} d^{4} x$ through the reorientation

$$
\exp \left\{\frac{1}{4} i \epsilon \Gamma_{m n} P_{m n}\right\}, \quad \epsilon \rightarrow 0 .
$$

It follows that six out of the 16 field equations (43) are redundant and follow from the 10 equations

$$
\begin{aligned}
& H_{\underline{\mu \nu}}=-\frac{1}{4} j_{5}{ } \kappa\left\langle\Psi_{/ \nu} \Gamma_{\mu} \Psi-\Psi \Gamma_{\mu} \Psi_{/ \nu}\right\rangle-\frac{1}{4} j_{5} *_{\kappa}\left\langle\Psi_{/ \mu} \Gamma_{\nu} \Psi-\Psi \Gamma_{\nu} \Psi_{/ \mu}\right\rangle \\
& -\frac{1}{2} \kappa\left(g_{m \mu} J_{m / \nu}+g_{m \nu} J_{m / \mu}\right)+g_{\mu \nu} L_{(s)} .
\end{aligned}
$$

Equations (47) and (48) allow a great deal of simplification in the field equations, since we can strike out all the terms of the form $T^{\rho}{ }_{\nu \rho}$ and $T^{\rho}{ }_{{ }_{\rho} \rho}$. Thus (44) and (45) become

$$
\begin{aligned}
& \kappa_{\mu}=\frac{1}{3} S_{\mu}-\frac{1}{2} B_{\mu \rho \sigma} F^{\rho \sigma}+\frac{1}{2} B_{0 \mu \rho} F^{0 \rho} \\
& \kappa J_{0}=\frac{3}{8} S_{0}-\frac{1}{4} A_{0 \rho \sigma} F^{\rho \sigma}
\end{aligned}
$$

where

$$
\begin{array}{rlrl}
S_{\mu} & =-F^{\rho}{ }_{\mu ; \rho}, & S_{0}=-F^{\rho}{ }_{0 ; \rho}, \\
\mathfrak{S}^{\mu}=\mathfrak{F}_{, \rho}^{\mu \rho}, & \mathfrak{S}^{0}=\mathfrak{F}_{, \rho}^{0 \rho} .
\end{array}
$$

Combining (31), (39) and (58) we obtain

$$
\begin{aligned}
\gamma B_{\mu \nu \lambda}= & \frac{1}{4} F_{\mu \nu ; \lambda}-\frac{1}{12}\left(S_{\mu} g_{\nu \lambda}-S_{\nu} g_{\mu \lambda}\right)+\frac{1}{2} A_{\mu \nu \rho} F_{\lambda}^{\rho} \\
& -\frac{1}{4}\left(F^{\rho}{ }_{\mu} A_{\nu \lambda \rho}-F^{\rho}{ }_{\nu} A_{\mu \lambda \rho}\right)+\frac{1}{4} F^{\rho \sigma}\left(A_{\mu \rho \sigma} g_{\nu \lambda}-A_{\nu \rho \sigma} g_{\mu \lambda}\right) \\
& +\frac{1}{2} A_{0 \mu \nu} F_{\lambda}^{0}-\frac{1}{4}\left(F^{0}{ }_{\mu} A_{0 \nu \lambda}-F^{0}{ }_{\nu} A_{0 \mu \lambda}\right)+\frac{1}{4} F^{\rho \sigma}\left(A_{0 \sigma \mu} g_{\mu \lambda}-A_{0 \sigma \nu} g_{\mu \lambda}\right) .
\end{aligned}
$$

Combining (33), (41) and (59) we get 


$$
\gamma B_{0 \lambda \mu}=\frac{1}{4} F_{0 \lambda ; \mu}-\frac{1}{16} S_{0} g_{\lambda \mu}+\frac{1}{4}\left(F_{\lambda}^{\rho}{ }_{\lambda} A_{0 \mu \rho}+F^{\rho}{ }_{\mu} A_{0 \lambda_{\rho}}\right)+\frac{1}{8} g_{\lambda \mu} A_{0 \rho \sigma} F^{\sigma \rho} .
$$

They satisfy the relations (47) and (48) identically.

The significance of equations (61) and (62) is that they eliminate $B_{\mu \nu \lambda}$ and $B_{0 \nu \lambda}$. In fact, we can regard now $A_{0}, A_{\mu}, A_{0 \mu \nu}, A_{\lambda \mu \nu}, A$ and $g_{\mu \nu}$ as the primary field quantities and $J_{0}, J_{\mu}, B_{0 \mu \nu}$ and $B_{\lambda \mu \nu}$ as derived quantities, their definition being (58), (59), (61) and (62) in conjunction with (60) and II. (23), II. (60). The perfect harmony of the primary field variables is best seen if we write

$$
A^{\rho \sigma}=E^{0 \mu \nu \rho \sigma} A_{0 \mu \nu}, \quad A^{\mu \nu \rho \sigma}=E^{0 \mu \nu \rho \sigma} A_{0},
$$

where $E^{0 \mu \nu \rho \sigma}$ is the tensor II. (9). The field is then described by a complete set of skew tensors $A, A_{\mu}, A_{\mu \nu}, A_{\mu \nu \rho}, A_{\mu \nu \rho \sigma}$ of gauge indices $1,0,-1,-2,-3$, respectively, and a symmetrical tensor $g_{\mu \nu}$ of gauge index -2 .

Defining now $T_{\mu \nu \lambda}, T_{0 \nu \lambda}, C_{\mu \nu}, D_{\mu \nu}, K_{\mu \nu}, R_{\mu \nu \rho \sigma}, R_{0 \sigma \mu \nu}, R_{\mu \nu}, R_{0 \nu}$ by equations (22), (29), (3), (4), (20), II. (45), II. (46), II. (68), II. (69), respectively, the field equations become

$$
\begin{aligned}
& \frac{1}{2} \Re^{0 v}{ }_{, \nu}=i \kappa j_{5} *\left\langle\Psi \Gamma^{0} \Psi\right\rangle, \\
& \Re_{, \nu}^{\mu \nu}=i \kappa j_{5} *\left\langle\Psi \Gamma^{\mu} \Psi\right\rangle \text {, } \\
& 2 \gamma A_{0 \lambda \mu}+\frac{1}{2}\left(F^{\rho}{ }_{\lambda} B_{0 \mu \rho}-F^{\rho}{ }_{\mu} B_{0 \lambda_{\rho}}\right)-\frac{1}{2} T_{\lambda \mu \rho} F^{\rho}{ }_{0} \\
& =-\frac{1}{4} j_{5}{ }^{*}\left\langle\Psi\left(\Gamma_{\lambda} \Gamma_{\mu} \Gamma_{0}-\Gamma_{0} \Gamma_{\mu} \Gamma_{\lambda}\right) \Psi\right\rangle, \\
& 2 \gamma A_{\mu \nu \lambda}-\frac{1}{4}\left(F_{\lambda}^{\rho}{ }_{\lambda} B_{\mu \nu \rho}+F^{\rho}{ }_{\mu} B_{\nu \lambda \rho}+F^{\rho}{ }_{\nu} B_{\lambda \mu \rho}\right)-\frac{1}{4}\left(F_{\lambda}^{0}{ }_{\lambda} A_{0 \mu \nu}+F^{0}{ }_{\mu} A_{0 \nu \lambda}+F^{0}{ }_{\nu} A_{0 \lambda \mu}\right) \\
& =-\frac{1}{8} j_{5}{ }^{*} \kappa\left\langle\Psi\left(\Gamma_{\lambda} \Gamma_{\mu} \Gamma_{\nu}-\Gamma_{\nu} \Gamma_{\mu} \Gamma_{\lambda}\right) \Psi\right\rangle, \\
& \text { (G) } \quad \frac{1}{4} F^{\rho \sigma}\left(R_{\mu \rho \sigma \nu}+R_{\nu \rho \sigma \mu}\right)-\frac{1}{4}\left(F^{\rho}{ }_{\nu} R_{\mu \rho}+F^{\rho \rho}{ }_{\mu} R_{\nu \rho}\right)+\frac{1}{2}\left(F^{\rho}{ }_{\nu} R_{\rho \mu}+F^{\rho}{ }_{\mu} R_{\rho \nu}\right) \\
& -\frac{1}{2} F^{\rho \sigma} R_{\rho \sigma} g_{\mu \nu}+\frac{1}{4} F^{0 \rho}\left(R_{\mu 0 \rho \nu}+R_{\nu 0 \rho \mu}\right)+\frac{1}{2}\left(F^{0}{ }_{\nu} R_{0 \mu}+F^{0}{ }_{\mu} R_{0 \nu}\right) \\
& -\frac{1}{2} F^{0 \sigma} R_{0 \sigma} g_{\mu \nu}+\gamma\left(R_{\mu \nu}+R_{\nu \mu}\right)-\gamma g^{\rho \sigma} R_{\rho \sigma} g_{\mu \nu}+\gamma \Lambda K_{\mu \nu} \\
& -\frac{1}{2} \gamma \Lambda g^{\rho \sigma} D_{\rho \sigma} g_{\mu \nu}-2 \gamma \epsilon_{5} A^{2} g_{\mu \nu}+\frac{1}{2} \kappa\left(J_{\mu ; \nu}+J_{\nu ; \mu}\right) \\
& +\frac{1}{2} \kappa J_{\rho}\left(B^{\rho}{ }_{\mu \nu}+B^{\rho}{ }_{\nu \mu}\right)+{ }^{\prime} J_{0} B^{0}{ }_{\mu \nu}-J^{\rho}{ }_{; \rho} g_{\mu \nu} \\
& =-\frac{1}{4} j_{5}{ }^{*}\left\langle\Psi_{/ \nu} \Gamma_{\mu} \Psi-\Psi \Gamma_{\mu} \Psi_{/ \nu}+\Psi_{/ \mu} \Gamma_{\nu} \Psi-\Psi \Gamma_{\nu} \Psi_{/ \mu}\right\rangle .
\end{aligned}
$$

The number of field equations is equal to the number of primary field variables viz. $1+4+6+4+1+10=26$.

Multiplication of (G) by $g^{\mu \nu}$ gives, with (55), (F0) and (F4), the relation

$$
\begin{aligned}
\frac{1}{2} F^{0 \rho} R_{0 \rho}+\frac{1}{2} A_{0} R_{; \nu}^{0 \nu}+2 \gamma g^{\rho \sigma} R_{\rho \sigma}+3 \kappa J_{; \rho}^{\rho}+2 \gamma \Lambda g^{\rho \sigma} D_{\rho \sigma} \\
-\gamma \Lambda g^{\rho \sigma} K_{\rho \sigma}+4 \gamma \epsilon_{5} A^{2}+\gamma \Lambda A^{-1} C_{; \mu ; \nu}^{\mu \nu}=0 .
\end{aligned}
$$

We shall write now equation (52) in a form which only involves real tensors 
as coefficients. Using the metrical derivatives of $\Psi$ as defined in II. (47), we have first

$$
\begin{aligned}
& \Gamma^{\mu} \Psi_{; \mu}-i A_{\mu} \Gamma^{\mu} \Psi-\frac{1}{4} i T_{m n \mu} \Gamma^{\mu} \Gamma_{m n} \Psi-\frac{1}{2} i S_{m 5 \mu} \Gamma^{\mu} \Gamma_{m 5} \Psi \\
&-i A_{0} \Gamma^{0} \Psi+\frac{1}{2} A j_{5} g \Psi=0 .
\end{aligned}
$$

Here

$$
\frac{1}{4} i T_{m n \mu} \Gamma^{\mu} \Gamma_{m n}=\frac{1}{4} \mathfrak{T}_{m n k} \Gamma_{k} \Gamma_{m} \Gamma_{n}=\frac{1}{4}\left(\mathfrak{A}_{m n k}+\mathfrak{B}_{m n k}\right) \Gamma_{k} \Gamma_{m} \Gamma_{n} .
$$

Now it follows from (47) that $B_{m n m}=0$, hence

$B_{m n k} \Gamma_{k} \Gamma_{m} \Gamma_{n}=\frac{1}{3} B_{m n k}\left(\Gamma_{k} \Gamma_{m} \Gamma_{n}+\Gamma_{m} \Gamma_{n} \Gamma_{k}+\Gamma_{n} \Gamma_{k} \Gamma_{m}\right)$

$$
=\frac{1}{3}\left(B_{m n k}+B_{n k m}+B_{k m n}\right) \Gamma_{k} \Gamma_{m} \Gamma_{n}=0
$$

by (25), and

$$
\frac{1}{4} i T_{m n \mu} \Gamma^{\mu} \Gamma_{m n}=\frac{1}{4} \mathfrak{A}_{m n k} \Gamma_{k} \Gamma_{m} \Gamma_{n}=\frac{1}{8} \mathfrak{A}^{\mu \nu \lambda}\left(\Gamma_{\mu} \Gamma_{\nu} \Gamma_{\lambda}-\Gamma_{\lambda} \Gamma_{\nu} \Gamma_{\mu}\right) .
$$

Similarly

$$
\frac{1}{2} i S_{m 5 \mu} \Gamma^{\mu} \Gamma_{m 5}=\frac{1}{2} g S_{m 5 k} \Gamma_{k} \Gamma_{m} \Gamma_{5}=\frac{1}{4} \mathfrak{Y}^{0 \mu \nu}\left(\Gamma_{\mu} \Gamma_{\nu} \Gamma_{0}-\Gamma_{0} \Gamma_{\nu} \Gamma_{\mu}\right) .
$$

Putting these into (65) we get the following "real" forms of the equation of motion:

$$
\begin{aligned}
\Gamma^{\mu} \Psi_{; \mu}-i A_{0} \Gamma^{0} \Psi-i A_{\mu} \Gamma^{\mu} \Psi-\frac{1}{8} \mathfrak{P}^{\mu \nu \lambda}\left(\Gamma_{\mu} \Gamma_{\nu} \Gamma_{\lambda}-\Gamma_{\lambda} \Gamma_{\nu} \Gamma_{\mu}\right) \Psi \\
-\frac{1}{2} \mathfrak{\mathcal { H } ^ { \mu \mu \nu }}\left(\Gamma_{\mu} \Gamma_{\nu} \Gamma_{0}-\Gamma_{0} \Gamma_{\nu} \Gamma_{\mu}\right) \Psi+\frac{1}{2} A j_{5} \mathfrak{g} \Psi=0 \\
\Psi_{; \mu}^{+} \Gamma^{\mu}+\Psi^{+} i A_{0} \Gamma^{0}+\Psi^{+} i A_{\mu} \Gamma^{\mu}+\frac{1}{8} \Psi^{+} \mathfrak{A}^{\mu \nu \lambda}\left(\Gamma_{\mu} \Gamma_{\nu} \Gamma_{\lambda}-\Gamma_{\lambda} \Gamma_{\nu} \Gamma_{\mu}\right) \\
+\frac{1}{4} \Psi^{+} \mathfrak{Q}^{0 \mu \nu}\left(\Gamma_{\mu} \Gamma_{\nu} \Gamma_{0}-\Gamma_{0} \Gamma_{\nu} \Gamma_{\mu}\right)-\frac{1}{2} \Psi^{+} j_{5} \mathfrak{g} A=0 .
\end{aligned}
$$

Or, using the tensors (63),

$$
\begin{aligned}
& \Gamma^{\mu} \Psi_{; \mu}+\left(\frac{1}{2} j_{5} \mathfrak{A}-i \mathfrak{\mathfrak { U } ^ { \mu }} \Gamma_{\mu}+\frac{1}{4} \mathfrak{U}^{\mu \nu} \Gamma_{\mu} \Gamma_{\nu}-\frac{1}{4} \mathfrak{A}^{\mu \nu \rho} \Gamma_{\mu} \Gamma_{\nu} \Gamma_{\rho}\right. \\
&\left.-\frac{1}{24} i \mathfrak{\mathfrak { U } ^ { \mu \nu \rho \sigma }} \Gamma_{\mu} \Gamma_{\nu} \Gamma_{\rho} \Gamma_{\sigma}\right) \Psi=0 .
\end{aligned}
$$

In this form the equation of motion involves only the primary field quantities as coefficients and has a perfectly symmetrical structure, provided that

$$
j_{5}=i \text {. }
$$

We shall see that this assumption also agrees with the physical interpretations given in the last section.

Multiplying (66) from the left by $-\kappa_{5}{ }^{*} \Psi^{+}=-\kappa\left(J_{5} \Psi\right)^{+}$and $\left(66^{+}\right)$from the right by $\kappa j_{5}{ }^{*} \Psi$ and forming the quarterspur, we get by (36), (37), (38), (40) and (42)

$$
\begin{aligned}
-\kappa j_{5} *\left\langle\Psi \Gamma^{\mu} \Psi_{; \mu}\right\rangle=\kappa j_{5} *\left\langle\Psi_{; \mu} \Gamma^{\mu} \Psi\right\rangle & \\
& =A_{0} \mathfrak{R}^{0}+A_{\mu} \mathfrak{R}^{\mu}+A_{0 \mu \nu} \mathfrak{R}^{\mu \nu 0}+A_{\mu \nu \lambda} \mathfrak{R}^{\lambda \mu \nu}+A \Omega .
\end{aligned}
$$

This can be regarded as the energy equation. 
It follows from (68) that

$$
\kappa j_{5} *\left\langle\Psi \Gamma^{\mu} \Psi_{; \mu}+\Psi_{; \mu} \Gamma^{\mu} \Psi\right\rangle=0 .
$$

Using a geodesic frame and orientation in which $\Gamma^{\mu}{ }_{\mu}=0$, this becomes the equation of continuity

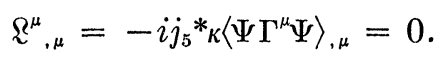

Unlike the energy equation which holds only in consequences of the field equations, (70) is an identity which, like (21), holds independently of the field equations. In fact, one can derive (70) by applying an infinitesimal phase transformation to the action integral which vanishes at the boundary. We have by II. (18)

$$
\delta A_{\mu}=\epsilon \lambda_{, \mu}
$$

which gives (70) immediately.

Further conservation identities can be derived by applying an infinitesimal transformation

$$
x_{\nu}=x_{\nu}{ }^{\prime}+\epsilon a^{\nu}\left(x^{\prime}\right)+O\left(\epsilon^{2}\right)
$$

to the $\mathcal{X}$-frame such that $a^{\nu}=0$ at the boundary. If $A^{\prime}{ }_{\mu}$ denotes the value of $A_{\mu}$ in the new frame then

$$
\begin{aligned}
A_{\mu}^{\prime}\left(x^{\prime}\right)=\sum_{\nu} \frac{\partial x_{\nu}}{\partial x_{\mu}^{\prime}} A_{\nu}(x) & =A_{\mu}(x)+\epsilon a_{, \mu}^{\nu} A_{\nu}(x)+O\left(\epsilon^{2}\right) \\
& =A_{\mu}\left(x^{\prime}\right)+\epsilon\left(A_{\mu, \nu} a^{\nu}+a_{, \mu}^{\nu} A_{\nu}\right)+O\left(\epsilon^{2}\right)
\end{aligned}
$$

hence, neglecting terms of order $\epsilon^{2}$,

$$
\delta A_{\mu}=\epsilon\left(A_{\mu, \nu} a^{\nu}+a^{\nu}{ }_{, \mu} A_{\nu}\right) .
$$

Similarly

$$
\begin{aligned}
& \delta S_{m 5 \mu}=\epsilon\left(S_{m 5 \mu, \nu} a^{\nu}+a^{\nu}{ }_{, \mu} S_{m 5 v}\right), \\
& \delta S_{m n \mu}=\epsilon\left(S_{m n \mu, \nu} a^{\nu}+a_{, \mu}^{\nu} S_{m n \nu}\right) \text {, } \\
& \delta g_{m}{ }^{\mu}=-\left(g_{m}{ }^{\mu}{ }_{\nu} a^{\nu}+a^{\mu}{ }_{,} g_{m}{ }^{\nu}\right),
\end{aligned}
$$

and we obtain from (13)

$$
\begin{aligned}
& \delta \int \mathfrak{R}_{(f)} d^{4} x=\epsilon \int\left\{\mathfrak{R}^{\mu}\left(A_{\mu, \nu} a^{\nu}+a^{\nu}{ }_{, \mu} A_{\nu}\right)+{\mathfrak{\mathfrak { R } ^ { \mu }}}_{m n}\left(S_{m n \mu, \nu} a^{\nu}+a^{\nu}{ }_{, \mu} S_{m n v}\right)\right. \\
& \left.+\mathfrak{2}^{\mu}{ }_{m 5}\left(S_{m 5 \mu, \nu} a^{\nu}+a^{\nu}{ }_{, \mu} S_{m 5 \nu}\right)-\mathfrak{S}_{m \mu}\left(g_{m}{ }^{\mu}{ }_{\nu} a^{\nu}+a^{\mu}{ }_{, \nu} g_{m}{ }^{\nu}\right)\right\} d^{4} x \\
& =\epsilon \int a^{\nu}\left\{\mathfrak{R}^{\mu} A_{\mu, \nu}-\left(\mathfrak{R}^{\mu} A_{\nu}\right)_{, \mu}+\mathfrak{R}^{\mu}{ }_{m n} S_{m n \mu, \nu}-\left(\mathfrak{R}^{\mu}{ }_{m n} S_{m n \nu}\right)_{, \mu}\right. \\
& \left.+\mathfrak{R}^{\mu}{ }_{m 5} S_{m 5 \mu, \nu}-\left(\mathfrak{R}^{\mu}{ }_{m 5} S_{m 5 \nu}\right)_{, \mu}-\mathfrak{S}_{m \mu} g_{m}{ }^{\mu}{ }_{, \nu}+\left(\mathfrak{S}_{m \nu} g_{m}{ }^{\mu}\right)_{, \mu}\right\} d^{4} x .
\end{aligned}
$$


The expression in the curled brackets must vanish identically since $\Omega_{(f)}$ is an invariant density, and we obtain

$$
\begin{aligned}
& \mathfrak{S}_{\nu}{ }^{\mu}{ }_{j \mu}=\mathfrak{R}^{\mu}{ }_{\mu} A_{\nu}-\mathfrak{R}^{\mu} F_{\mu \nu}+\left(\mathfrak{Q}^{\mu \rho \sigma} T_{\rho \sigma \nu}\right)_{; \mu}-\mathfrak{R}^{\mu \rho \sigma} T_{\rho \sigma \mu ; \nu} \\
& -\left(\mathbb{R}^{\mu \rho 0} T_{0 \rho \nu}\right)_{; \mu}+\mathfrak{Q}^{\mu \rho 0} T_{0 \rho \mu ; \nu} .
\end{aligned}
$$

The same conservation identities can be shown to hold for the corresponding spinor tensors (36)-(43).

Remark. If $\Psi$ is a homogeneous spinor field which satisfies the field equations and the equation of motion and $\eta$ is a constant unitary element, then, by I. (36), $\Psi \eta$ also satisfies the equations. $\Psi$ and $\Psi \eta$ must be regarded as equivalent solutions which represent identical geometrical situations.

10. Cosmological Models. Although the field equations and equations of motion have been derived from a variation principle, there is nothing to show that the equations admit exact solutions with a non-vanishing spinor field. The purpose of this section is to show that such solutions exist; at the same time it will be possible to determine the value (or at least the most probable value) of the constant $\Lambda$. For the rest of the paper it will be assumed that $j_{5}=i$, $\epsilon_{5}=-1$, in accordance with (67).

To obtain a particularly simple "cosmological" solution, we assume that all the primary field components vanish with the exception of $g_{\mu \nu}$ and $A$ (which, as we know, cannot vanish). Then also the secondary field quantities vanish except $R_{\rho \mu \nu \sigma}=G_{\rho \mu \nu \sigma}$ and $C_{\mu}$, and the field equations become

$$
\begin{gathered}
\left\langle\Psi \Gamma^{0} \Psi\right\rangle=0, \\
\left\langle\Psi \Gamma^{\mu} \Psi\right\rangle=0, \\
\left\langle\Psi\left(\Gamma_{\lambda} \Gamma_{\mu} \Gamma_{0}-\Gamma_{0} \Gamma_{\mu} \Gamma_{\lambda}\right) \Psi\right\rangle=0, \\
\left\langle\Psi\left(\Gamma_{\lambda} \Gamma_{\mu} \Gamma_{\nu}-\Gamma_{\nu} \Gamma_{\mu} \Gamma_{\lambda}\right) \Psi\right\rangle=0, \\
\Lambda T^{2} C_{; \mu \nu}^{\mu \nu}+4 T^{-1}=-\frac{1}{2} \kappa \gamma^{-1}\langle\Psi \Psi\rangle, \\
G_{\mu \nu}-\frac{1}{2} G g_{\mu \nu}+\frac{1}{2} \Lambda K_{\mu \nu}-\frac{1}{4} \Lambda D g_{\mu \nu}+T^{-2} g_{\mu \nu}=-\frac{1}{2} \kappa \gamma^{-1} T_{\mu \nu}
\end{gathered}
$$

where $G=g^{\rho \sigma} G_{\rho \sigma}, D=C^{\rho \sigma} C_{\rho \sigma}, C_{\rho \sigma}=T_{, \rho ; \sigma}$, and

$$
\begin{aligned}
K_{\mu \nu} & =-C_{\mu} C_{\nu ; \rho}{ }^{\rho}-C_{\nu} C_{\mu ; \rho}^{\rho}+C_{\mu \nu} C_{\rho}{ }^{\rho}+C^{\rho} C_{\mu \nu ; \rho}, \\
T_{\mu \nu} & =\frac{1}{4 i}\left\langle\Psi_{; \nu} \Gamma_{\mu} \Psi-\Psi \Gamma_{\mu} \Psi_{; \nu}+\Psi_{; \mu} \Gamma_{\nu} \Psi-\Psi \Gamma_{\nu} \Psi_{; \mu}\right\rangle .
\end{aligned}
$$

The equation of motion takes the form

$$
\Gamma^{\mu} \Psi_{; \mu}+\frac{1}{2} i T^{-1} \mathrm{~g} \Psi=0 .
$$

For the rest of this section we agree that roman and Greek suffixes shall 
not take the value 4, except the suffix $\tau$ which will denote a Greek 4 (to distinguish it from a roman 4 ). The same applies to the summation convention.

For the line element we take ${ }^{21}$

$$
g_{\mu \nu}=-t^{2 \alpha} \delta_{\mu \nu}, \quad g_{\mu \tau}=0, \quad g_{\tau \tau}=1
$$

where $t=x_{4}$ and $\alpha$ is a positive constant. We also assume that $d T / d t=\beta$ is a constant, so that by taking a suitable origin for time reckoning, we have

$$
T=\beta t .
$$

The only non-vanishing Christoffel symbols are

$$
\left\{\begin{array}{l}
\tau \\
\mu \nu
\end{array}\right\}=-\alpha t^{-1} g_{\mu \nu}, \quad\left\{\begin{array}{c}
\mu \\
\tau \nu
\end{array}\right\}=\alpha t^{-1} \delta_{\mu \nu}
$$

They give

$$
\begin{gathered}
G_{\mu \nu}=\alpha(3 \alpha-1) t^{-2} g_{\mu \nu}, \quad G_{\mu \tau}=0, \quad G_{\tau \tau}=3 \alpha(\alpha-1) t^{-2}, \quad G=6 \alpha(2 \alpha-1) t^{-2}, \\
C_{\mu}=0, \quad C_{\tau}=\beta, \quad C_{\mu \nu}=\alpha \beta t^{-1} g_{\mu \nu}, \quad C_{\tau \mu}=C_{\tau \tau}=0, \\
C_{\mu ; \nu}+C_{\mu ; \tau}{ }^{\nu}=0, \quad C^{\mu}=3 \alpha \beta t^{-1}, \quad C_{\tau}{ }^{\tau}=0, \\
{ }^{\nu}+C_{\tau ; \tau}{ }^{\tau}=-3 \alpha^{2} \beta t^{-2}, \\
C_{\mu \nu ; \tau}=-\alpha \beta t^{-2} g_{\mu \nu}, \quad C_{\tau \nu ; \tau}=C_{\tau \tau ; \tau}=0, \\
C_{; \mu ; \nu}+C^{\mu \tau}{ }_{; \mu ; \tau}+C^{\tau \mu} ; \tau ; \mu \\
K_{\mu \nu}=\alpha(3 \alpha-1) C^{2} t^{-2} g_{\mu \nu}, \quad K_{\tau \mu}=0, \quad K_{\tau \tau}=6 \alpha^{2} \beta^{2} t^{-2}, \quad D=-3 \alpha^{2}(3 \alpha-2) \beta t^{-3}, \quad D \alpha^{2} \beta^{2} t^{-2} .
\end{gathered}
$$

Hence by (76) and (77)

$$
\begin{gathered}
\left(4 \beta^{-1}+3 \Lambda \beta^{3} \alpha^{2}(3 \alpha-2)\right) t^{-1}=-\frac{1}{2} \kappa \gamma^{-1}\langle\Psi \Psi\rangle, \\
{\left[\alpha(3 \alpha-2)\left(\frac{1}{4} \Lambda \beta^{2}-1\right)+\beta^{-2}\right] t^{-2} g_{\mu \nu}=-\frac{1}{2} \kappa \gamma^{-1} T_{\mu \nu},} \\
T_{\mu \tau}=0 \\
\left(\frac{9}{4} \alpha^{2} \Lambda \beta^{2}-3 \alpha^{2}+\beta^{-2}\right) t^{-2}=-\frac{1}{2} \kappa \gamma^{-1} T_{\tau \tau} .
\end{gathered}
$$

These are satisfied if

$$
\Lambda \beta^{2}=\frac{4}{3 \alpha+1}, \quad 3 \alpha^{2} \beta^{2}=\frac{3 \alpha+1}{3 \alpha-2},
$$

and

$$
\begin{gathered}
\langle\Psi \Psi\rangle=0, \\
T_{\mu \nu}=T_{\mu \tau}=T_{\tau \tau}=0 .
\end{gathered}
$$

${ }^{21}$ This is the line element of the simplest cosmological model which satisfies the field equations with $\Psi=0$, see $[11]$. 
In order to satisfy (81) we assume that with a suitable orientation of the spinor frames ${ }^{22}$

$$
g_{n \mu}=\frac{1}{i} t^{\alpha} \delta_{n \mu}, \quad g_{n \tau}=g_{4 \mu}=0, \quad g_{4 \tau}=1 .
$$

They give, with (83) and II. (38),

$$
Q_{m n \nu}=Q_{m 4 \nu}=Q_{m 4 \tau}=0, \quad Q_{m 4 \nu}=-\alpha t^{-1} g_{m \nu},
$$

hence, by II. (47),

$$
\Psi_{; \mu}=\Psi_{, \mu}+\frac{1}{2} \alpha t^{-1} g_{m \mu} \Gamma_{m} \Gamma_{4} \Psi, \quad \Psi_{; \tau}=\Psi_{, \tau},
$$

and equation (80) becomes

$$
g_{k}{ }^{\mu} \Gamma_{k} \Psi_{, \mu}+\Gamma_{4} \Psi_{, \tau}+\frac{3}{2} \alpha t^{-1} \Gamma_{4} \Psi+\frac{1}{2} i \beta^{-1} t^{-1} \Psi=0 .
$$

Let us set tentatively

$$
\Psi=\left(a+b \Gamma_{4}\right)\left(1+\Gamma_{5}\right)
$$

where $a$ and $b$ are functions of $t$ alone. Then

$$
\Psi^{+}=\left(1-\Gamma_{5}\right)\left(a^{*}+b^{*} \Gamma_{4}\right),
$$

and all of the equations (72)-(75) and (89), (90) are satisfied. (93) and (94) give

$$
t \frac{d a}{d t}+\frac{3}{2} \alpha a+\frac{1}{2} i \beta^{-1} b=0, \quad t \frac{d b}{d t}+\frac{3}{2} \alpha b+\frac{1}{2} i \beta^{-1} a=0,
$$

a solution of which is

$$
\begin{aligned}
& a=t^{-\frac{3}{2} \alpha}\left(c_{1} t^{i / 2 \beta}+c_{2} t^{-i / 2 \beta}\right), \\
& b=t^{-\frac{3}{2} \alpha}\left(-c_{1} t^{i / 2 \beta}+c_{2} t^{-i / 2 \beta}\right)
\end{aligned}
$$

for arbitrary constants $c_{1}, c_{2}$. It shows that the (physically significant) quantity

$$
g^{\frac{1}{2}} \Psi=\left\{\left(c_{1} t^{i / 2 \beta}+c_{2} t^{-i / 2 \beta}\right)+\left(-c_{1} t^{i / 2 \beta}+c_{2} t^{-i / 2 \beta}\right) \Gamma_{4}\right\}\left(1+\Gamma_{5}\right)
$$

is periodic in $\log t$ with period $4 \beta \pi$.

We have not yet disposed of the value of $\Lambda$; in fact, any positive value of $\Lambda$ is compatible with a model (81), where the value of $\alpha$ is determined from (88). Undoubtedly the most satisfactory value is $\alpha=1$ which corresponds to the linearly expanding cosmological model

$$
g_{\mu \nu}=-T^{2} \delta_{\mu \nu}, \quad g_{\mu \tau}=0, \quad g_{\tau \tau}=1 .
$$

It gives

$$
\Lambda \beta^{2}=1, \quad \beta^{2}=\frac{4}{3},
$$

hence

$$
\Lambda=\frac{3}{4} \text {. }
$$

22 The phase frame has already been fixed by the assumption $A_{\mu}=0, A_{\tau}=0$. 
11. Physical Interpretations. It is impossible at this stage to give more than a brief outline of the possible physical interpretation of the various quantities which appear in the field equations. In particular, we shall refrain from identifying the spinor field $\Psi$ with anything physical, for the very good reason that it does not belong to the real space and therefore it has to be eliminated from the ultimate numerical description of any given physical situation.

It is fairly clear from the cosmological solution that the interpretation of the metric tensor should be the same as in general relatively, namely a quantity which describes the gravitational field. If this interpretation is accepted then $A$ becomes HubBLE's recession "constant", and $T$ must be interpreted as cosmic time, representing the epoch at which the universe is considered.

In order to obtain an interpretation for the other primary field quantities, it is best to turn to the equation of motion (66). This is of Dirac's type and incorporates every conceivable type of interaction. The most familiar terms are those involving $A_{0}, A_{\mu}$ and $A$, namely

$$
-i A_{0} \Gamma^{0}, \quad-i A_{\mu} \Gamma^{\mu}, \quad \frac{1}{2} i A \Psi .
$$

The first and second terms suggest, by analogy with Dirac's equation, ${ }^{23}$ that $A_{\mu}$ should represent the electromagnetic vector potential and $A_{0}$ (or $A_{\mu \nu \rho \sigma}$ ) the pseudo-scalar meson potential. The third term shows that the inertial mass of the electron is equal to the recession constant, hence is inversely proportional to the age of the universe.

The interpretation of the remaining terms in (66) is not so clear; presumably $A_{0 \mu \nu}$ represents a pseudo-tensor meson field and $A_{\mu \nu \lambda}$ a kind of "spin field".

Of the quantities at the right of the field equations (F0)-(F4), expression (F1) is the most familiar; it represents the electrical current density. The equation reveals the surprising fact that $\Re^{\mu \nu}$, and not $\mathfrak{F}^{\mu \nu}$, must be regarded as the electromagnetic field density whose divergence gives the current density. To find out the relation between the two, let us write down the approximate form of the field equations under the assumption that the field is weak. We make the following assumptions:

(1) There exists a world frame which is very nearly Galilean over a finite region of $R$, and the direction of the $x_{4}$-axis very nearly coincides with the direction of the gradient of $T$. Or, using again the convention of the previous section on Greek suffixes, we assume that

$$
\begin{aligned}
& g_{\mu \nu}=-\delta_{\mu \nu}+h_{\mu \nu}, \quad g_{\mu \tau}=h_{\mu \tau}, \quad g_{\tau \tau}=1+h_{\tau \tau}, \\
& C_{\mu}=\beta \varphi_{, \mu}, \quad C_{\tau}=\beta\left(1+\varphi_{, \tau}\right)
\end{aligned}
$$

where $\beta=2 / \sqrt{3}$ and $h_{\mu \nu}, h_{\mu \tau}, h_{\tau \tau}, \varphi$ are small.

(2) All the other primary field quantities are very small when measured with the absolute gauge $\gamma=1$.

${ }^{23}$ The units are chosen so that $c=1, h / 2 \pi=1$, and $e^{-1} A_{\mu}$ is the electromagnetic potential in Heavyside units. 
Neglecting products of small quantities and reverting to the old conventions on Greek suffixes, we get from (58)-(62)

$$
\begin{aligned}
\gamma B_{\mu \nu \lambda} & =\frac{1}{4} F_{\mu \nu, \lambda}-\frac{1}{12}\left(S_{\mu} g_{\nu \lambda}-S_{\nu} g_{\mu \lambda}\right), \\
\gamma B_{0 \lambda \mu} & =\frac{1}{4} F_{0 \lambda, \mu}-\frac{1}{16} S_{0} g_{\lambda \mu}, \\
\kappa J_{\mu} & =\frac{1}{3} S_{\mu}, \quad \kappa J_{0}=\frac{3}{8} S_{0} .
\end{aligned}
$$

They give, since $S^{\mu}{ }_{, \mu}=0$,

$$
J_{, \mu}^{\mu}=0,
$$

and, by II. (68), II. (69),

$$
\begin{aligned}
& R_{\mu \nu}=G_{\mu \nu}+A_{\mu \nu, \rho}^{\rho}-\frac{1}{6 \gamma} S_{\mu, \nu}, \\
& R_{0 \nu}=A_{0 \nu, \rho}^{\rho}-\frac{3}{16 \gamma} S_{0, \nu} .
\end{aligned}
$$

Putting these into (F0)-(F4) and $\mathrm{G}$, we get the following approximate field equations:

$$
\begin{gathered}
\square S^{0}=-\frac{32}{3} \kappa \gamma\left\langle\Psi \Gamma^{0} \Psi\right\rangle, \\
\square S^{\mu}=-12 \kappa \gamma\left\langle\Psi \Gamma^{\mu} \Psi\right\rangle, \\
A_{0 \lambda \mu}=\frac{1}{8} i \kappa \gamma^{-1}\left\langle\Psi\left(\Gamma_{\lambda} \Gamma_{\mu} \Gamma_{0}-\Gamma_{0} \Gamma_{\mu} \Gamma_{\lambda}\right) \Psi\right\rangle, \\
A_{\mu \nu \lambda}=\frac{1}{16} i \kappa \gamma^{-1}\left\langle\Psi\left(\Gamma_{\lambda} \Gamma_{\mu} \Gamma_{0}-\Gamma_{\nu} \Gamma_{\mu} \Gamma_{\lambda}\right) \Psi\right\rangle, \\
\square \square \varphi=\frac{1}{2} \frac{\partial G}{\partial x_{4}}, \\
G_{\mu \nu}-\frac{1}{2} G g_{\mu \nu}+\frac{3}{8} K_{\mu \nu}=\frac{1}{8} i \kappa \gamma^{-1}\left\langle\Psi_{, \nu} \Gamma_{\mu} \Psi-\Psi \Gamma_{\mu} \Psi_{, \nu}+\Psi_{, \mu} \Gamma_{\nu} \Psi-\Psi \Gamma_{\nu} \Psi_{, \mu}\right\rangle
\end{gathered}
$$

where $\square$ is the d'Alembertian $g^{\mu \nu}\left(\partial / \partial x_{\mu}\right)\left(\partial / \partial x_{v}\right)$. It is an operator of gauge index 2.

Equations $\left(\mathrm{F}^{*}\right)$ and $\left(\mathrm{G}^{*}\right)$ were obtained in [11], except for the matter term on the right of $\left(\mathrm{G}^{*}\right)$. Equation $\left(\mathrm{F} 1^{*}\right)$ shows that the true current vector is not $S^{\mu}$, as postulated by MAxweLl, but $\square S^{\mu}$. Hence $\mathfrak{S}^{\mu}$ takes over the role of the vector potential density, it automatically satisfies the Lorentz condition $\Im^{\mu}{ }_{, \mu}=0$. If the phase frame is normalised by the Lorentz condition $A^{\mu}{ }_{, \mu}=0$, then the left hand side of $\left(\mathrm{F} 1^{*}\right)$ can be written in the form $\square \square A^{\mu}$. Similarly the left hand side of $\left(\mathrm{F}^{*}\right)$ is equal to $\square \square A^{0}$.

(36), (37), (102), $\left(\mathrm{F} 0^{*}\right)$ and $\left(\mathrm{F} 1^{*}\right)$ give the relations

$$
\square J^{0}=4 L^{0}, \quad \square J^{\mu}=4 L^{\mu}
$$

between the two current vectors $J^{\alpha}$ and $L^{\alpha}$, the first one being connected with the $\phi$ component and the second one with the $\Psi$ component of the Hamiltonian 
spinor field. It appears from (106) that the substance current $L^{0}, L^{\mu}$ is a source of "photon current" waves $J^{0}, J^{\mu}$.

Equations $\left(\mathrm{F}^{*}\right)$ and $\left(\mathrm{F} 3^{*}\right)$ are, in this approximate form, not field equations in the proper sense, but merely defining relations for $A_{0 \lambda \mu}$ and $A_{\mu \nu \lambda}$. These quantities enter only as second order non-linear correction terms in the rest of the field equations, and consequently their effect is not likely to be significant, except perhaps in nuclear processes where the field variables might attain large values. It is hardly surprising that so far they have not been considered as independent field quantities.

\section{References.}

[1] Blade, W. L. \& Jehle, H., An introduction to spinors, Rev. Mod. Phys. 25, 714-28, 1953.

[2] Börner, H., Darstellungen von Gruppen, Springer, Berlin, 1955.

[3] Chevaluex, C. C., The algebriac theory of spinors, Columbia University Press, New York, 1954.

[4] Eddingron, A. S., Relativity theory of protons and electrons, Cambridge University Press, 1936.

[5] Fock, V., Geometrisierung der Diracschen Theorie des Electrons, Z. Phys. 57, 261-77, 1929.

[6] Hlavatry, V., The spinor connection in the unified Einstein theory of relativity, Proc. Nat. Acad. Sci. U. S. A., 39, 501-6, 1953.

[7] Ingraham, R. L. Spinor relativity, Il Nuovo Cimento (9) 10, 27-42, 1953.

[8] RIEsz, M., Sur certaines notions fondamentales en théorie quantique relativiste, Dixième Congrès des Mathematiciens Scandinaves, Copenhague, 1946.

[9] SchröDinger, E., Space-Time Structure, Cambridge University Press, 1950.

[10] Stemnrod, N., The topology of fibre bundles, Princeton University Press, 1951.

[11] Szekeres, G., New formulation of the general theory of relativity, Phys. Rev. 97, 212-23, 1955.

[12] Kantor, W. \& Szekeres, G., Cosmic time and the field equations of general relativity, Phys. Rev. 104, 831-4, 1956.

The University of Adelaide, Adelaide, South Australia. 